\title{
Fabric Tensor Characterization of Tensor-Valued Directional Data: Solution, Accuracy, and Symmetrization
}

\author{
Kuang-dai Leng and Qiang Yang \\ State Key Laboratory of Hydroscience and Engineering, Tsinghua University, Beijing 100084, China \\ Correspondence should be addressed to Qiang Yang, yangq@mails.tsinghua.edu.cn
}

Received 21 April 2012; Revised 5 July 2012; Accepted 19 July 2012

Academic Editor: Luis Javier Herrera

Copyright (c) 2012 K.-d. Leng and Q. Yang. This is an open access article distributed under the Creative Commons Attribution License, which permits unrestricted use, distribution, and reproduction in any medium, provided the original work is properly cited.

Fabric tensor has proved to be an effective tool statistically characterizing directional data in a smooth and frame-indifferent form. Directional data arising from microscopic physics and mechanics can be summed up as tensor-valued orientation distribution functions (ODFs). Two characterizations of the tensor-valued ODFs are proposed, using the asymmetric and symmetric fabric tensors respectively. The later proves to be nonconvergent and less accurate but still an available solution for where fabric tensors are required in full symmetry. Analytic solutions of the two types of fabric tensors characterizing centrosymmetric and anticentrosymmetric tensor-valued ODFs are presented in terms of orthogonal irreducible decompositions in both two- and threedimensional (2D and 3D) spaces. Accuracy analysis is performed on normally distributed random ODFs to evaluate the approximation quality of the two characterizations, where fabric tensors of higher orders are employed. It is shown that the fitness is dominated by the dispersion degree of the original ODFs rather than the orders of fabric tensors. One application of tensor-valued ODF and fabric tensor in continuum damage mechanics is presented.

\section{Introduction}

Many quantities arising from microscopic physics and mechanics are directional dependent, and as is often the case, are randomly and discontinuously distributed along the orientations. Statistical characterization of such directional data is essential [1-4]. With regard to physical problems, such characterization must take a frame-indifferent form, or a tensorial form which is invariant to coordinate transformations; see, for example, Kanatani [5], Advani and Tucker [6], and Yang et al. [7].

Directional data can be generalized as orientation distribution functions (ODFs). In its classical definition $[3-5,8,9]$, an ODF is actually a probability density function of a 
continuous random orientational variable, which practically represents the fraction of the total number of directional elements per unit stereo-angle along a certain direction. The classically defined ODFs can be abstracted as scalar-valued directional functions, because from a vector-space viewpoint, their values on each orientation are nothing but normalized scalars. Therefore, the classical ODFs are referred to as scalar-valued ODFs in this paper, following Yang et al. [7], in regard to the generality of tensor-valued ODFs.

The scalar-valued ODFs can be statistically characterized by their fabric tensors $[5$, 6, 9-21]. Such tensors are labeled fabric because they were first and have been extensively introduced to describe the fabric (or texture) of structured solid materials, such as granular [5, 10-12] and microcracked [13-16] media, polycrystals and polymers [17, 18], and bones [19], and prove to be an effective tool bridging the multiscale behaviors of the materials. Recent applications of fabric tensors extend beyond material studies to wherever directional variables are focused, such as mixed fluids $[6,20]$ and medical imaging $[9,21]$, whether being called fabric tensors or not. The analytic solutions of fabric tensors of higher orders are obtained by Kanatani [5], who characterized the scalar-valued ODFs in expansions of three kinds of fabric tensors and addressed their equivalence to the Fourier series expansion in $2 \mathrm{D}$ and to the spherical harmonics expansion in 3D. Yang et al. [22] showed that fabric tensors of different orders are not independent: fabric tensors of lower orders can be precisely determined by those of higher orders.

The concept of the tensor-valued ODFs is relatively new, but their objectivity in physics and mechanics is visible. Yang et al. $[7,23]$ proved that the damage effective stress in damage mechanics is inherently a vector-valued ODF, which cannot be fully described by a Cauchylike stress tensor of order two. In fabric-tensor-based micromechanics of granular media; see, for example, Rahmoun et al. [11] and $\mathrm{Li}$ and $\mathrm{Yu}$ [12], the contact forces and contact vectors should both be taken for vector-valued ODFs. In the microplane theory of Bažant and Gambarova [24] and Carol and Bažant [25], the microplane elasticity tensor is essentially a second-order tensor-valued ODF that defines a one-to-one linear map between the stress and strain vector-valued ODFs. The fabric tensor algebra of vector-valued ODFs is preliminarily studied by Yang et al. [7], who derived the explicit expressions of symmetric fabric tensors up to the sixth order. $\mathrm{Li}$ and $\mathrm{Yu}$ [12] provided a stepwise repetitive method to determine asymmetric fabric tensors of higher orders. However, general analytic solution is not found in previous studies.

The approximation quality of the fabric tensor characterization is not so frequently stressed in previous studies, perhaps because there are few alternative ways to characterize directional data in a tensorial form. Kanatani [5] suggested the test of uniformity to evaluate the fitness. Li and $\mathrm{Yu}$ [12] simply defined the proximity index for both scalar- and vectorvalued ODFs. One can intuitively estimate the accuracy of the zeroth-, second-, and fourthorder fabric tensor approximations to randomly distribute scalar-valued ODFs in many of the referred studies $[5,6,9,11,13,14,16]$. Nevertheless, systematic accuracy analysis has not been reported yet, even for scalar-valued ODFs.

In this paper, we complete the fabric tensor algebra for the largest generality of tensorvalued ODFs. A strict but asymmetric characterization is presented in complete parallel to that of the scalar-valued ODFs by Kanatani [5]. The relationship of fabric tensors of different orders [22] holds for the tensor-valued ODFs in this case. However, the asymmetric fabric tensors may be inconvenient for practical use because of potential inconsistency with physical hypotheses and observations. Therefore, a nonstandard symmetric characterization is proposed, which is proved to be nonconvergent but still available for most irregular tensor-valued ODFs. Analytic solutions of the two types of fabric tensors characterizing 
centrosymmetric and anticentrosymmetric tensor-valued ODFs in 2D and 3D are presented in the forms of orthogonal irreducible decompositions by Zheng and Zou [26]. Accuracy analysis is performed on normally distributed random ODFs to evaluate the approximation quality of the two characterizations, where fabric tensors of higher orders are employed.

\section{Notation and Operation of Tensor and Orientation}

In this paper, we mainly use the abstract notation of tensors to simplify the expressions. For this purpose, quite a few notations and operators must be predefined. The abstract form of a generic tensor is written in bold and nonitalic, with its order shown in the subscript, for example, a fourth-order tensor $\mathbf{T}_{4}=\left\{T_{i j k l}\right\}$ (Cartesian components in \{\} ). In particular, scalars are written like $\psi$ and vectors like $\mathbf{v}=\left\{v_{i}\right\}$.

\subsection{Tensor Symmetrization}

In the component notation, the symmetrization of a generic tensor $T_{i_{1} i_{2} \cdots i_{n} j_{1} j_{2} \cdots j_{m}}$ on the indices $i_{1} i_{2} \cdots i_{n}$ is denoted by $T_{\bar{i}_{1} i_{2} \cdots i_{n}} j_{1} j_{2} \cdots j_{m}$, which is defined as the sum of the components of $\mathbf{T}_{n+m}$ indexed by the $n$ ! permutations of the overbared subscripts $\overline{i_{1} i_{2} \cdots i_{n}}$ divided by $n$ ! If two symmetrizations are required at the same time (luckily, two is enough for our use), double overbars must be used. Here are two examples:

$$
T_{i \overline{j k l} l}=\frac{1}{2}\left(T_{i j k l}+T_{i k j l}\right), \quad R_{\bar{i} \overline{\bar{p}}} T_{\bar{j} \overline{\bar{q}}}=\frac{1}{4}\left(R_{i p} T_{j q}+R_{j p} T_{i q}+R_{i q} T_{j p}+R_{j q} T_{i p}\right) .
$$

In the abstract notation, the symmetrization is simply represented by the overbared orders, for example, $\mathbf{T}_{\bar{n} \oplus m}=\left\{T_{\overline{i_{1} i_{2} \cdots i_{n}} j_{1} j_{2} \cdots j_{m}}\right\}, \mathbf{T}_{1 \oplus \overline{2} \oplus 1}=\left\{T_{i \overline{j k l}}\right\}$, and $\mathbf{R}_{\overline{1} \oplus \overline{\overline{1}}} \otimes \mathbf{T}_{\overline{1} \oplus \overline{\overline{1}}}=\left\{R_{\bar{i} \overline{\bar{p}}} T_{\bar{j} \overline{\bar{q}}}\right\}$. The operator $\oplus$ means sequenced addition of orders, which removes ambiguities when the orders are operated. For example, $\mathbf{T}_{1 \oplus \overline{2} \oplus 1}$ precisely indicates that the second and third orders are symmetrized, while $\mathbf{T}_{1+\overline{2}+1}$ may be mistaken for $\mathbf{T}_{2+\overline{2}}$ or $\mathbf{T}_{\overline{2}+2}$.

Full symmetrization of a tensor $\mathbf{T}_{n}$ can be realized by defining the alternating product operation signified by $\diamond$,

$$
\mathbf{T}_{\bar{n}} \stackrel{\text { def }}{=} \mathbf{1}_{\overline{1} \oplus 1}^{\otimes n} \stackrel{n}{\diamond} \mathbf{T}_{n}=\mathbf{1}_{1 \oplus \overline{1}}^{\otimes n} \stackrel{n}{\otimes} \mathbf{T}_{n}
$$

where $\mathbf{1}_{2}=\left\{\delta_{i j}\right\}$ is the second-order identity tensor and $\mathbf{1}_{\overline{1} \oplus 1}^{\otimes n}$ means the $n$th power tensor product of $\mathbf{1}_{\overline{1} \oplus 1}$ :

$$
\mathbf{1}_{\overline{1} \oplus 1}^{\otimes n} \stackrel{\text { def }}{=} \underbrace{\mathbf{1}_{\overline{1} \oplus 1} \bigotimes \mathbf{1}_{\overline{1} \oplus 1} \bigotimes \cdots \otimes \mathbf{1}_{\overline{1} \oplus 1}}_{n \text { times }} .
$$

The alternating product is actually a modified inner product in which the left-hand operand tensor participates on alternate orders. Equation (2.2) makes itself explicit in the component form

$$
T_{\overline{i_{1} i_{2} \cdots i_{n}}}=\delta_{\overline{i_{1} j_{1}}} \delta_{\overline{i_{2}} j_{2}} \cdots \delta_{\overline{i_{n}} j_{n}} T_{j_{1} j_{2} \cdots j_{n}} .
$$




\subsection{Tensor Deviatorization and Orthogonal Irreducible Decomposition}

In this subsection, we only consider full symmetric tensors. Successive contraction of a full symmetric tensor $\mathbf{T}_{\bar{n}}$ for $m$ times is denoted by

$$
\mathbf{T}_{\overline{n \ominus 2 m}} \stackrel{\text { def }}{=}\left\{T_{\overline{i_{1} i_{2} \cdots i_{n-2 m}} j_{1} j_{1} j_{2} j_{2} \cdots j_{m} j_{m}}\right\} .
$$

A symmetric tensor $\mathbf{D}_{\bar{n}}$ is termed a deviator tensor if it is traceless, $\mathbf{D}_{\bar{n} \cdot 2}=0$. The deviatorization operation of a symmetric tensor means to segregate its deviator part, as signified by the operator $\llbracket \cdot \rrbracket$ on its order,

$$
\begin{gathered}
\mathbf{T}_{\overline{\llbracket 2 n \rrbracket}} \stackrel{\text { def }}{=} \sum_{r=0}^{n} \kappa_{2 n}^{r} \mathbf{T}_{\overline{2 n \ominus 2 r}} \bigotimes \mathbf{I}_{\overline{2 r}}, \\
\mathbf{T}_{\overline{\llbracket 2 n+1 \rrbracket}} \stackrel{\text { def }}{=} \sum_{r=0}^{n} \kappa_{2 n+1}^{r} \mathbf{T}_{\overline{2 n+1 \ominus 2 r}} \bigotimes \mathbf{I}_{\overline{2 r}},
\end{gathered}
$$

where $\mathbf{I}_{2 r}$ is the $2 r$ th order identity tensor, $\mathbf{I}_{2 r}=\mathbf{1}_{\overline{2}}^{\otimes r}$. The coefficients $\kappa_{m}^{r}$ are determined in such a way that $\kappa_{m}^{0} \stackrel{\text { def }}{=} 1$ and any contraction of (2.6a) and (2.6b) vanishes. The results of $\kappa_{m}^{r}$ are provided by Kanatani [5],

$$
\kappa_{m}^{r}= \begin{cases}\frac{(-1)^{r}}{2^{2 r}} \frac{m}{m-r}\left(\begin{array}{c}
m-r \\
r
\end{array}\right), & \text { in 2D, } \\
\frac{(-1)^{r}\left(\begin{array}{c}
m \\
2 r
\end{array}\right)\left(\begin{array}{c}
m-1 \\
r
\end{array}\right)}{\left(\begin{array}{c}
2 m-1 \\
2 r
\end{array}\right)}, & \text { in 3D, }\end{cases}
$$

where $m$ is the order of $\mathbf{T}$, whether even or odd. For examples, the deviatorizations of the 3D symmetric tensors of orders from 1 to 4 are shown in the component forms

$$
\begin{aligned}
& T_{\llbracket i \rrbracket}=T_{i}, T_{\llbracket i j \rrbracket}=T_{i j}-\frac{1}{3} T_{r r} \delta_{i j}, \\
& T_{\llbracket i j k \rrbracket}=T_{i j k}-\frac{3}{5} T_{r r i} \delta_{\overline{j k}}, \quad T_{\llbracket i j k l \rrbracket}=T_{i j k l}-\frac{6}{7} T_{r r i j} \delta_{\overline{k l}}+\frac{3}{35} T_{r r s s} I_{\overline{i j k l}} .
\end{aligned}
$$

The orthogonal irreducible decomposition theorem of symmetric tensors of higher orders is proposed by Zheng and Zou [26], which states that any symmetric tensor $\mathbf{T}_{\bar{m}}$ in either 2D or 3D can be orthogonally decomposed into deviator-tensor-induced irreducible bases in the following unified forms:

$$
\begin{gathered}
\mathbf{T}_{\overline{2 n}}=\sum_{r=0}^{n} \mathbf{D}_{\overline{2 r}} \bigotimes \mathbf{I}_{\overline{2 n-2 r},} \\
\mathbf{T}_{\overline{2 n+1}}=\sum_{r=0}^{n} \mathbf{D}_{\overline{2 r+1}} \bigotimes \mathbf{I}_{\overline{2 n-2 r},}
\end{gathered}
$$


where $\mathbf{D}_{\overline{2 r}}$ and $\mathbf{D}_{\overline{2 r+1}}$ are two clusters of deviator tensors. The deviatorization of any deviatortensor-induced irreducible basis vanishes except for the last term where $r=n$, namely, $\mathbf{D}_{\overline{\llbracket 2 r}} \otimes \mathbf{I}_{\overline{2 n-2 r \rrbracket}}=0(r=0,1, \ldots, n-1)$ and $\mathbf{D}_{\llbracket \overline{\llbracket n \rrbracket}}=\mathbf{D}_{\overline{2 n}}$.

\subsection{Definition and Integral of Orientation}

Any orientation about a point can be defined by a unit vector $\mathbf{n}$ starting at that point and ending on a unit circle denoted $S^{1}$ in 2D or a unit sphere denoted $S^{2}$ in 3D,

$$
\mathbf{n} \stackrel{\text { def }}{=} \begin{cases}\{\cos \phi, \sin \phi\}, & \text { in } 2 \mathrm{D}, \\ \{\sin \theta \cos \phi, \sin \theta \sin \phi, \cos \theta\}, & \text { in } 3 \mathrm{D},\end{cases}
$$

where $\phi$ and $\theta$ are the stereo angles defining the orientation $\mathbf{n}$.

The contour integral $\oint(\cdot) \mathrm{d} \mathbf{n}$ in 2D and 3D are, respectively, defined as

$$
\oint(\cdot) \mathrm{d} \mathbf{n} \stackrel{\text { def }}{=} \begin{cases}\frac{1}{2 \pi} \oint_{S^{1}}(\cdot) \mathrm{d} l=\frac{1}{2 \pi} \int_{0}^{2 \pi}(\cdot) \mathrm{d} \phi, & \text { in 2D, } \\ \frac{1}{4 \pi} \oint_{S^{2}}(\cdot) \mathrm{d} A=\frac{1}{4 \pi} \int_{0}^{\pi} \int_{0}^{2 \pi}(\cdot) \sin \theta \mathrm{d} \phi \mathrm{d} \theta, & \text { in 3D. }\end{cases}
$$

Noting that $2 \pi$ and $4 \pi$ are the circumference of $S^{1}$ and the spherical area of $S^{2}$, respectively, so the integral $\oint(\cdot) \mathrm{d} \mathbf{n}$ represents an average value of the distribution $(\cdot)$ over all orientations.

The identity tensors are related to the integral of orientations by

$$
\mathbf{I}_{2 n}=\mathbf{1}_{\overline{2}}^{\otimes n}=\alpha_{2 n} \oint \mathbf{n}^{\otimes 2 n} \mathrm{~d} \mathbf{n}=\alpha_{2 n} \oint \mathbf{N}_{2 n}(\mathbf{n}) \mathrm{d} \mathbf{n},
$$

where $\mathbf{N}_{2 n}(\mathbf{n}) \stackrel{\text { def }}{=} \mathbf{n} \otimes 2 n$ and the coefficient $\alpha_{2 n}$ is given by

$$
\alpha_{2 n}= \begin{cases}\frac{2^{2 n}}{\left(\begin{array}{c}
2 n \\
n
\end{array}\right)}, & \text { in 2D, } \\
2 n+1, & \text { in 3D. }\end{cases}
$$

Noting that $\mathbf{n} \cdot \mathbf{n}=1$, the successive contraction of $\mathbf{I}_{2 n}$ for $n-r$ times takes the form

$$
\mathbf{I}_{2 n \ominus 2(n-r)}=\frac{\alpha_{2 n}}{\alpha_{2 r}} \mathbf{I}_{\overline{2 r}} .
$$

For example, in 3D, $\delta_{i j}=3 \oint n_{i} n_{j} \mathrm{~d} \mathbf{n}, I_{i j k l}=5 \oint n_{i} n_{j} n_{k} n_{l} \mathrm{~d} \mathbf{n}$, and $I_{i j k k}=(3 / 5) \delta_{i j}$.

\section{Asymmetric Characterization of Tensor-Valued ODFs}

A tensor-valued ODF is a tensor function dependent on the predefined orientation $\mathbf{n}$. A generic tensor-valued ODF of order $m$ is denoted by $\mathbf{t}_{m}=\mathbf{t}_{m}(\mathbf{n})$, which may be randomly 
and discontinuously distributed along the orientations. The fabric tensor characterization first requires that $\mathbf{t}_{m}(\mathbf{n})$ be decomposed into the centrosymmetric and anticentrosymmetric parts namely, $\mathbf{t}_{m}(\mathbf{n})=\mathbf{s}_{m}(\mathbf{n})+\mathbf{a}_{m}(\mathbf{n})$, where

$$
\begin{aligned}
\mathbf{s}_{m}(\mathbf{n})=\frac{1}{2}\left[\mathbf{t}_{m}(\mathbf{n})+\mathbf{t}_{m}(-\mathbf{n})\right], & \mathbf{s}_{m}(\mathbf{n})=\mathbf{s}_{m}(-\mathbf{n}), \\
\mathbf{a}_{m}(\mathbf{n})=\frac{1}{2}\left[\mathbf{t}_{m}(\mathbf{n})-\mathbf{t}_{m}(-\mathbf{n})\right], & \mathbf{a}_{m}(\mathbf{n})=-\mathbf{a}_{m}(-\mathbf{n}) .
\end{aligned}
$$

We first consider the centrosymmetric tensor-valued ODF $\mathbf{s}_{m}(\mathbf{n})$. Let $\mathbf{s}_{m}(\mathbf{n})$ be approximated by another tensor-valued ODF $\boldsymbol{s}_{m}(\mathbf{n})$, which is precisely determined by a tensor $\mathbf{S}_{m+2 n}$ (even orders must be added due to the centrosymmetry of $\mathbf{s}_{m}(\mathbf{n})$ ),

$$
\mathbf{S}_{m}(\mathbf{n}) \approx \mathbf{s}_{m}(\mathbf{n}) \stackrel{\text { def }}{=} \mathbf{S}_{m+2 n} \stackrel{2 n}{n} \mathbf{N}_{2 n}(\mathbf{n})
$$

for example, $\mathbf{s}_{2}(\mathbf{n}) \approx \boldsymbol{s}_{2}(\mathbf{n}) \stackrel{\text { def }}{=} \mathbf{S}_{4}{ }^{2} \cdot(\mathbf{n} \otimes \mathbf{n})$. Because $\mathbf{N}_{2 n}(\mathbf{n})$ is full symmetric, it is quite visible that $\mathbf{S}_{m+2 n}=\mathbf{S}_{m \oplus \overline{2 n}}$.

The tensor $\mathbf{S}_{m+2 n}$ is defined as the fabric tensor of the second kind (or simply referred to as fabric tensor where no ambiguity arises) of the tensor-valued ODF $\mathbf{s}_{m}(\mathbf{n})$ if the following condition of least-square minimization of error is imposed:

$$
\Delta \stackrel{\text { def }}{=} \oint\left|\mathbf{s}_{m}(\mathbf{n})-\boldsymbol{s}_{m}(\mathbf{n})\right|^{2} \mathrm{~d} \mathbf{n}, \quad \frac{\partial \Delta}{\partial \mathbf{S}_{m+2 n}}=0
$$

Applied with (3.2), (3.3) yields

$$
\oint \mathbf{s}_{m}(\mathbf{n}) \bigotimes \mathbf{N}_{2 n}(\mathbf{n}) \mathrm{d} \mathbf{n}=\oint \mathbf{s}_{m}(\mathbf{n}) \bigotimes \mathbf{N}_{2 n}(\mathbf{n}) \mathrm{d} \mathbf{n},
$$

which can be further recast as

$$
\mathbf{S}_{m+2 n}{ }^{2 n} \cdot \mathbf{I}_{4 n}=\alpha_{4 n} \boldsymbol{s}_{m \oplus \overline{2 n}}
$$

where $\boldsymbol{s}_{m \oplus \overline{2 n}}$ is defined as the fabric tensor of the first kind of $\mathbf{s}_{m}(\mathbf{n})$,

$$
\boldsymbol{s}_{m \oplus \overline{2 n}} \stackrel{\text { def }}{=} \oint \mathbf{s}_{m}(\mathbf{n}) \bigotimes \mathbf{N}_{2 n}(\mathbf{n}) \mathrm{d} \mathbf{n}
$$

One contracting property of $s_{m \oplus \overline{2 n}}$ is favorable, $s_{m \oplus \overline{2 n \ominus 2}}=s_{m \oplus \overline{2 n-2}}$.

Such asymmetric characterization of tensor-valued ODFs is the intrinsic extension of that of scalar-valued ODFs by Kanatani [5], in that the components of the tensor-valued ODF are handled as independent scalar-valued ODFs, whose fabric tensor expansion proves to be equivalent to the Fourier series expansion in $2 \mathrm{D}$ and to the spherical harmonics expansion in 3D. In other words, the asymmetric characterization is convergent; namely, the 
approximation error $\Delta$ is a nonincreasing function of the used order $2 n$ and tends to zero when $2 n$ tends to infinity.

\subsection{Analytic Solution of $S_{m+2 n}$}

Evidently, $\boldsymbol{s}_{m} \oplus \overline{2 n}$ can be approximately determined by observed values of $\mathbf{s}_{m}(\mathbf{n})$ on finite numbers of orientations, so what left for us to do is to find the explicit expression of $\mathbf{S}_{m+2 n}$ with respect to $\delta_{m \oplus \overline{2 n}}$, or the inversed form of (3.5).

Resorting to (2.9a), the orthogonal irreducible decomposition of $\mathbf{S}_{m+2 n}$ takes the following form:

$$
\mathbf{S}_{m+2 n}=\mathbf{S}_{m \oplus \overline{2 n}} \stackrel{\text { def }}{=} \sum_{r=0}^{n} \boldsymbol{\Phi}_{m \oplus \overline{2 r}} \bigotimes \mathbf{I}_{\overline{2 n-2 r}}
$$

where $\boldsymbol{\Phi}_{m} \oplus \overline{2 r}(r=0,1, \ldots, n)$ are defined by [5] as the fabric tensors of the third kind of $\mathbf{s}_{m}(\mathbf{n})$, which are, however, barely perceptible in practical use.

Applying (3.7) to (3.5), and noting that any contraction of $\Phi_{m \oplus \overline{2 r}}$ vanishes, one obtains

$$
\sum_{r=0}^{n} \frac{\xi_{2 n, 2 r}^{2 r}}{\alpha_{2 n+2 r}} \Phi_{m} \oplus \overline{2 r} \bigotimes \mathbf{I}_{\overline{2 n-2 r}}=\boldsymbol{s}_{m \oplus \overline{2 n}}
$$

where the coefficient $\xi_{2 n, 2 r}^{2 r}$ is determined by (if one substitutes (3.7) to (3.5) and observes the result, he may realize that $\xi_{2 n, 2 r}^{2 s}$ is all about a ball and box puzzle. Assume that $2 n+2 r$ distinct balls, $2 n$ colored red and $2 r$ blue, are paired and placed into $n+r$ sequenced boxes, each box with a pair. And $\xi_{2 n, 2 r}^{2 s}$ is actually the probability of that $2 s$ boxes are filled with a pair in different colors)

$$
\xi_{2 n, 2 r}^{2 s}=2^{2 s} \frac{\left(\begin{array}{c}
n+r \\
2 s
\end{array}\right)\left(\begin{array}{c}
n+r-2 s \\
n-s
\end{array}\right)}{\left(\begin{array}{c}
2 n+2 r \\
2 r
\end{array}\right)}
$$

Successively contracting (3.8) for $n-t$ times on the symmetrized orders, taking in the property $\boldsymbol{\delta}_{m \oplus \overline{2 n \ominus 2}}=\boldsymbol{\delta}_{m \oplus \overline{2 n-2}}$,

$$
\sum_{r=0}^{t} \frac{\xi_{2 n, 2 r}^{2 r} \xi_{2 n, 2 r}^{2 t}}{\alpha_{2 n+2 r}} \Phi_{m \oplus \overline{2 r}} \bigotimes \mathbf{I} \overline{2 t-2 r}=\delta_{m} \oplus \overline{2 t},
$$

where $\zeta_{2 n, 2 r}^{2 t}$ is the product of the coefficients arising from each contraction,

$$
\zeta_{2 n, 2 r}^{2 t}=\prod_{s=0}^{n-t-1} \frac{\left[\left(\left(\alpha_{2 n-2 s-2 r}\right) /\left(\alpha_{2 n-2 s-2 r-2}\right)\right)\left(\begin{array}{c}
2 n-2 s-2 r \\
2
\end{array}\right)+\left(\begin{array}{c}
2 n-2 s-2 r \\
1
\end{array}\right)\left(\begin{array}{c}
2 r \\
1
\end{array}\right)\right]}{\left(\begin{array}{c}
2 n-2 s \\
2
\end{array}\right)} .
$$

Taking the deviatorization of (3.10) on the remaining symmetric orders, noting that any term in the left-hand side vanishes except the last one, one finally acquires the fabric tensors of the third kind of $\mathbf{s}_{m}(\mathbf{n})$,

$$
\boldsymbol{\Phi}_{m \oplus \overline{2 t}}=\frac{\alpha_{2 n+2 t}}{\xi_{2 n, 2 t}^{2 t} \zeta_{2 n, 2 t}^{2 t}} \boldsymbol{s}_{m \oplus \overline{\llbracket 2 t \rrbracket}}=\eta_{2 n}^{2 t} s_{m \oplus \overline{\llbracket 2 t \rrbracket}} .
$$


The coefficient $\eta_{2 n}^{2 t}$ is fully simplified by taking in (2.13), which turns out to be independent of $n$,

$$
\eta^{2 t}= \begin{cases}2^{2 t}, & \text { in 2D, } \\
\frac{4 t+1}{2^{2 t}}\left(\begin{array}{l}
4 t \\
2 t
\end{array}\right), & \text { in 3D. }\end{cases}
$$

The analytic solution of fabric tensors of the second kind of $\mathbf{s}_{m}(\mathbf{n})$ is finally obtained by applying (3.12) and (2.6a) to (3.7),

$$
\mathbf{S}_{m \oplus \overline{2 n}}=\sum_{t=0}^{n} \eta^{2 t} \sum_{r=0}^{t} \kappa_{2 t}^{r} \boldsymbol{s}_{m \oplus \overline{2 t-2 r}} \bigotimes \mathbf{I}_{\overline{2 n-2 t+2 r}}
$$

For the anticentrosymmetric tensor-valued ODF $\mathbf{a}_{m}(\mathbf{n})$, odd orders must be appended to the fabric tensors of the second kind,

$$
\mathbf{a}_{m}(\mathbf{n}) \approx a_{m}(\mathbf{n}) \stackrel{\text { def }}{=} \mathbf{A}_{m+2 n+1} \stackrel{2 n+1}{N_{2 n+1}}(\mathbf{n})
$$

Accordingly, the fabric tensors of the first kind are defined as

$$
\mathcal{A}_{m} \oplus \overline{2 n+1} \stackrel{\text { def }}{=} \oint \mathbf{a}_{m}(\mathbf{n}) \bigotimes \mathbf{N}_{2 n+1}(\mathbf{n}) \mathrm{d} \mathbf{n}
$$

The analytic solution of $\mathbf{A}_{m+2 n+1}$ with respect to $\mathcal{A}_{m} \oplus \overline{2 n+1}$ can be acquired completely parallel to that of $\mathbf{S}_{m+2 n}$, which takes the following form:

$$
\mathbf{A}_{m \oplus \frac{2 n+1}{2 n}}=\sum_{t=0}^{n} \eta^{2 t+1} \sum_{r=0}^{t} \kappa_{2 t+1}^{r} \mathcal{A}_{m} \oplus \overline{2 t+1-2 r} \bigotimes \mathbf{I}_{2 n-2 t+2 r}
$$

where $\eta^{2 t+1}$ is calculated just by replacing $2 t$ with $2 t+1$ in (3.13).

For the generic tensor-valued ODF $\mathbf{t}_{m}(\mathbf{n})$, its centrosymmetric part $\mathbf{s}_{m}(\mathbf{n})$ and anticentrosymmetric part $\mathbf{a}_{m}(\mathbf{n})$ are characterized independently, which means that the order of $\mathbf{A}$ is unnecessarily one larger than that of $\mathbf{S}$ (anyway, we take this assumption in the accuracy analysis). For quick reference, the ultimately solved coefficients of the fabric tensors of the second kind up to the sixth and seventh approximation orders are listed in Table 1.

\subsection{Relationship between Fabric Tensors of Different Orders}

Assume that two fabric tensors $\mathbf{S}_{m \oplus \overline{2 n_{1}}}$ and $\mathbf{S}_{m \oplus \overline{2 n_{2}}}$, where $n_{1}<n_{2}$, are used to characterize the centrosymmetric tensor-valued ODF $\mathbf{s}_{m}(\mathbf{n})$

$$
\begin{aligned}
& \mathbf{S}_{m}(\mathbf{n}) \approx \mathbf{s}_{m}^{1}(\mathbf{n})=\mathbf{S}_{m \oplus \overline{2 n_{1}}} \stackrel{2 n_{1}}{\cdot} \mathbf{N}_{2 n_{1}}(\mathbf{n}) \\
& \mathbf{s}_{m}(\mathbf{n}) \approx \mathbf{s}_{m}^{2}(\mathbf{n})=\mathbf{S}_{m \oplus \overline{2 n_{2}}}{ }^{2 n_{2}} \cdot \mathbf{N}_{2 n_{2}}(\mathbf{n})
\end{aligned}
$$


Table 1: Coefficients of lower order fabric tensors of the second kind using asymmetric characterization for tensor-valued ODFs (2D and 3D).

\begin{tabular}{|c|c|c|c|c|c|c|c|c|c|}
\hline $\mathbf{s}_{m}(\mathbf{n})$ & $s_{m}$ & $\boldsymbol{s}_{m \oplus^{2}}$ & $\delta_{m \oplus \overline{4}}$ & $\boldsymbol{s}_{m \oplus \overline{6}}$ & $\mathbf{a}_{m}(\mathbf{n})$ & $\mathcal{A}_{m \oplus \overline{1}}$ & $\mathcal{A}_{m \oplus \overline{3}}$ & $\mathcal{A}_{m \oplus \overline{5}}$ & $\mathcal{A}_{m \oplus \overline{7}}$ \\
\hline $\mathbf{S}_{m}$ & 1,1 & & & & $\mathbf{A}_{m \oplus \overline{1}}$ & 2,3 & & & \\
\hline $\mathbf{S}_{m \oplus \overline{2}}$ & $-1,-\frac{3}{2}$ & $4, \frac{15}{2}$ & & & $\mathbf{A}_{m \oplus \overline{3}}$ & $-4,-\frac{15}{2}$ & $8, \frac{35}{2}$ & & \\
\hline $\mathbf{S}_{m \oplus \overline{4}}$ & $1, \frac{15}{8}$ & $-12,-\frac{105}{4}$ & $16, \frac{315}{8}$ & & $\mathbf{A}_{m \oplus \overline{5}}$ & $6, \frac{105}{8}$ & $-32,-\frac{315}{4}$ & $32, \frac{693}{8}$ & \\
\hline $\mathbf{S}_{m \oplus \overline{\bar{b}}}$ & $-1,-\frac{35}{16}$ & $24, \frac{945}{16}$ & $-80,-\frac{3465}{16}$ & $64, \frac{3003}{16}$ & $\mathbf{A}_{m \oplus \overline{7}}$ & $-8,-\frac{315}{16}$ & $80, \frac{3465}{16}$ & $-192,-\frac{9009}{16}$ & $128, \frac{6435}{16}$ \\
\hline
\end{tabular}

In view of (3.4), the fabric tensors of the first kind defined by (3.6) take the following form:

$$
\begin{aligned}
& s_{m \oplus \overline{2 n_{1}}} \stackrel{\text { def }}{=} \oint \mathbf{s}_{m}(\mathbf{n}) \bigotimes \mathbf{N}_{2 n_{1}}(\mathbf{n}) \mathrm{d} \mathbf{n}=\oint \boldsymbol{s}_{m}^{1}(\mathbf{n}) \bigotimes \mathbf{N}_{2 n_{1}}(\mathbf{n}) \mathrm{d} \mathbf{n}, \\
& \boldsymbol{s}_{m \oplus \overline{2 n_{2}}} \stackrel{\text { def }}{=} \oint \mathbf{s}_{m}(\mathbf{n}) \bigotimes \mathbf{N}_{2 n_{2}}(\mathbf{n}) \mathrm{d} \mathbf{n}=\oint \boldsymbol{s}_{m}^{2}(\mathbf{n}) \bigotimes \mathbf{N}_{2 n_{2}}(\mathbf{n}) \mathrm{d} \mathbf{n} .
\end{aligned}
$$

The following relation between $\boldsymbol{s}_{m \oplus \overline{2 n_{1}}}$ and $\boldsymbol{s}_{m \oplus \overline{2 n_{2}}}$ is evident:

$$
\boldsymbol{s}_{m \oplus \overline{2 n_{1}}}=\oint \mathbf{s}_{m}(\mathbf{n}) \bigotimes \mathbf{N}_{2 n_{1}}(\mathbf{n}) \mathrm{d} \mathbf{n}=\oint \mathbf{s}_{m}^{2}(\mathbf{n}) \bigotimes \mathbf{N}_{2 n_{1}}(\mathbf{n}) \mathrm{d} \mathbf{n},
$$

where the second equality is achieved by contracting (3.19b) for $n_{2}-n_{1}$ times.

Equation (3.20) indicates that once a fabric tensor $\mathbf{S}_{m \oplus \overline{2 n_{2}}}$ of the original ODF $\mathbf{s}_{m}(\mathbf{n})$ is obtained, those of lower orders $\mathbf{S}_{m \oplus \overline{2 n_{1}}}$ can be precisely determined by the approximate ODF $\boldsymbol{s}_{m}^{2}(\mathbf{n})$ induced from $\mathbf{S}_{m \oplus \overline{2 n_{2}}}$, needless to use the original ODF again. Simply put, fabric tensors of lower orders can be precisely determined by those of higher orders. Such dependence implies that fabric tensors of different orders cannot be treated as independent state variables in thermodynamics [22].

\subsection{Accuracy Analysis}

The approximation quality of the fabric tensor characterization is quantified by the squared error ratio $\varepsilon^{n}$ defined as

$$
\varepsilon^{n} \stackrel{\text { def }}{=} \frac{\Delta^{n}}{\oint\left|\mathbf{t}_{m}(\mathbf{n})\right|^{2} \mathrm{~d} \mathbf{n}}=\frac{\oint\left|\mathbf{t}_{m}(\mathbf{n})-\boldsymbol{t}_{m}^{n}(\mathbf{n})\right|^{2} \mathrm{~d} \mathbf{n}}{\oint\left|\mathbf{t}_{m}(\mathbf{n})\right|^{2} \mathrm{~d} \mathbf{n}},
$$

where the superscript $n$ determines the approximation orders applied for both the centrosymmetric and anticentrosymmetric parts of $\mathbf{t}_{m}(\mathbf{n})$,

$$
\mathbf{t}_{m}(\mathbf{n}) \approx \boldsymbol{t}_{m}^{n}(\mathbf{n})=\mathbf{S}_{m \oplus} \overline{2 n}^{2 n} \mathbf{N}_{2 n}(\mathbf{n})+\mathbf{A}_{m} \overline{2 n+1}^{2 n+1} \cdot \mathbf{N}_{2 n+1}(\mathbf{n})
$$

Accuracy analysis performed on scalar-valued ODFs is sufficient to evaluate the fitness of the asymmetric characterization, because each component of the tensor-valued ODFs 
is characterized in terms of independent scalar-valued ODFs. Nevertheless, vector-valued ODFs are investigated in the next section to calibrate the accuracy of the symmetric characterization.

The original scalar-valued ODF $\psi(\mathbf{n})$ is assigned on each direction with a random real number sampling from the normal distribution with mean $\mu$ and standard deviation $\sigma$, denoted $N\left(\mu, \sigma^{2}\right)$. In the accuracy analysis, $\mu$ is fixed at 1.0 and $\sigma$ ranges from 0.1 to 0.5 with increment 0.1 . The order parameter $n$ ranges from 0 to 5 , namely, as high as the tenth, and eleventh fabric tensors are involved. The squared error ratios in $2 \mathrm{D}$ and $3 \mathrm{D}$ are shown in Figures 1 and 2, respectively.

As evidenced by the figures, the increase of $\sigma$ will to a considerable extent reduce the accuracy when the approximation order is at a lower level, for example, the mostly used second and fourth-order $(n=1,2)$. The loss of accuracy can be remedied by increasing the order to a higher level such as the tenth order $(n=5)$, which, however, cannot be effective due to upsurging computational expense. Therefore, the approximation quality of the fabric tensor characterization is dominated by the dispersion degree of the original ODFs rather than the orders of fabric tensors. The original and approximate ODFs related to distribution $N\left(1.0,0.3^{2}\right)$ in $2 \mathrm{D}$ and $3 \mathrm{D}$ are plotted in Figures 3 and 4 , respectively, from which the fitness can be intuitively estimated.

\section{Symmetric Characterization of Tensor-Valued ODFs}

For quite a few non-scalar-valued ODFs in practice, their values on all orientations are objectively symmetric, that is, $\mathbf{t}_{m}(\mathbf{n})=\mathbf{t}_{\bar{m}}(\mathbf{n})$. Such circumstances include all the vector-valued ODFs and many second-order tensor-valued ODFs in physics, such as the elasticity ODF $E_{i j}(\mathbf{n})=E_{j i}(\mathbf{n})$ and the conservative diffusion gradient ODF $G_{i j}(\mathbf{n})=G_{j i}(\mathbf{n})$. The symmetry of the fabric tensors of such ODFs is obvious, $\mathbf{S}_{m+2 n}=\mathbf{S}_{\overline{\bar{m}} \oplus \overline{2 n}}$ and $\mathbf{A}_{m+2 n+1}=\mathbf{A}_{\overline{\bar{m}} \oplus \overline{2 n+1}}$. On many occasions, however, these fabric tensors are supposed to be fully symmetrized to keep consistency with physical hypotheses or observations. Therefore, the symmetric characterization is proposed, where the fabric tensors are fully symmetrized.

Consider again that the centrosymmetric part of $\mathbf{t}_{\bar{m}}(\mathbf{n})$ denoted $\mathbf{s}_{\bar{m}}(\mathbf{n})$, which is characterized by the symmetrized fabric tensor of the second kind $\mathbf{S}_{\overline{m+2 n}}$,

$$
\mathbf{S}_{\bar{m}}(\mathbf{n}) \approx \mathbf{s}_{\bar{m}}(\mathbf{n}) \stackrel{\text { def }}{=} \mathbf{S}_{\overline{m+2 n}} \stackrel{2 n}{ } \mathbf{N}_{2 n}(\mathbf{n})
$$

The condition of least-square minimization of error goes as follows:

$$
\Delta \stackrel{\text { def }}{=} \oint\left|\mathbf{s}_{\bar{m}}(\mathbf{n})-\mathbf{s}_{\bar{m}}(\mathbf{n})\right|^{2} \mathrm{~d} \mathbf{n}, \quad \frac{\partial \Delta}{\partial \mathbf{S}_{m+2 n}}=0 .
$$

Noting that $\mathbf{S}_{\overline{m+2 n}}$ is related to $\mathbf{S}_{m+2 n}$ by the alternating product defined in (2.2), (4.2) can be recast as

$$
\oint s_{\bar{m}}(\mathbf{n}) \bigotimes \mathbf{N}_{\overline{2 n}}(\mathbf{n}) \mathrm{d} \mathbf{n}=\oint \mathbf{s}_{\bar{m}}(\mathbf{n}) \bigotimes \mathbf{N}_{\overline{2 n}}(\mathbf{n}) \mathrm{d} \mathbf{n},
$$




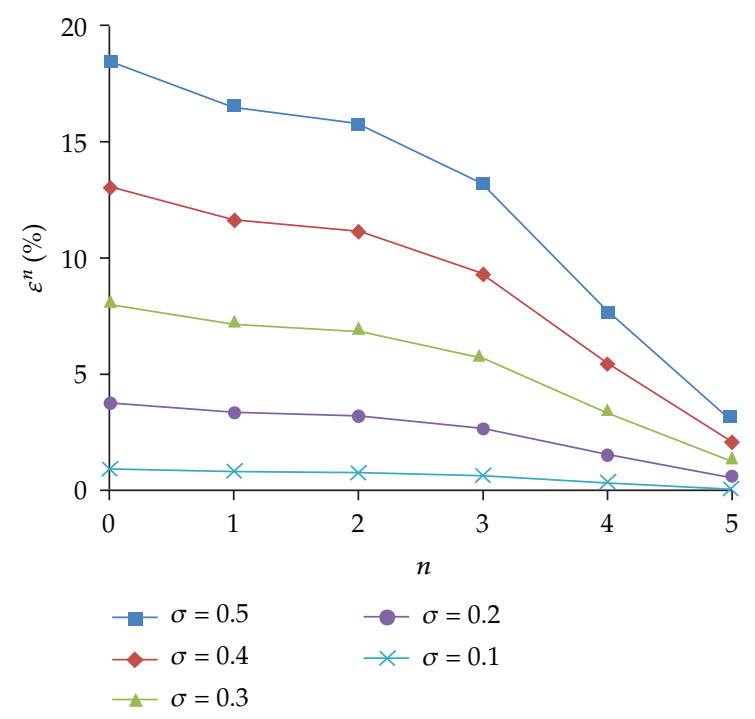

Figure 1: Squared error ratios of asymmetric characterizations of scalar-valued ODFs sampling from $N\left(1.0, \sigma^{2}\right)$ in $2 \mathrm{D}$.

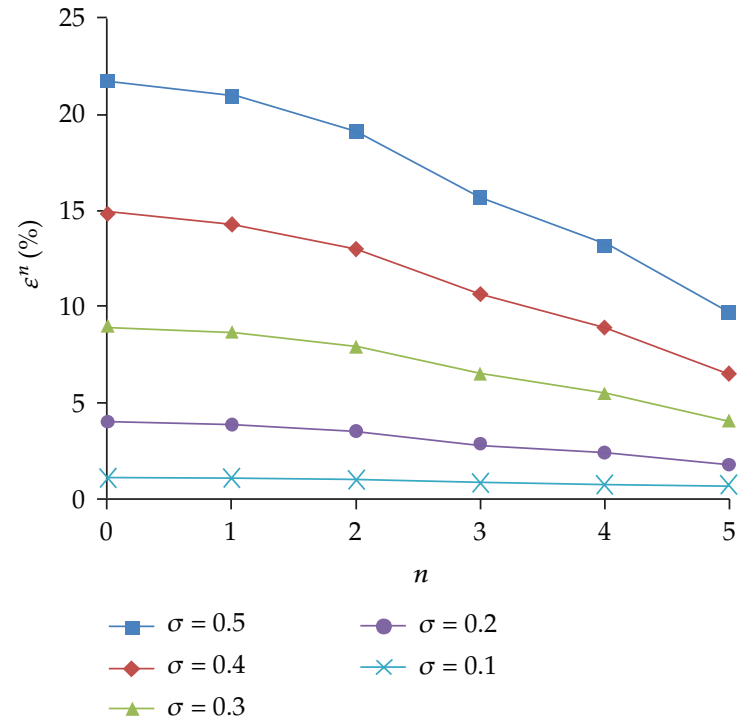

Figure 2: Squared error ratios of asymmetric characterizations of scalar-valued ODFs sampling from $N\left(1.0, \sigma^{2}\right)$ in $3 \mathrm{D}$.

which can be further reduced to

$$
\mathbf{1}_{\overline{1} \oplus \overline{\overline{1}}}^{\otimes m} \stackrel{m}{\diamond} \mathbf{S}_{m+2 n}{ }^{2 n} \cdot \mathbf{I}_{\overline{2 n} \oplus \overline{\overline{2 n}}}=\alpha_{4 n} \boldsymbol{\delta}_{\overline{m+2 n}}
$$

where $\delta_{\overline{m+2 n}}$ is termed the symmetrized fabric tensor of the first kind,

$$
\S_{\overline{m+2 n}} \stackrel{\text { def }}{=} \oint \mathbf{s}_{\bar{m}}(\mathbf{n}) \bigotimes \mathbf{N}_{\overline{2 n}}(\mathbf{n}) \mathrm{d} \mathbf{n} .
$$




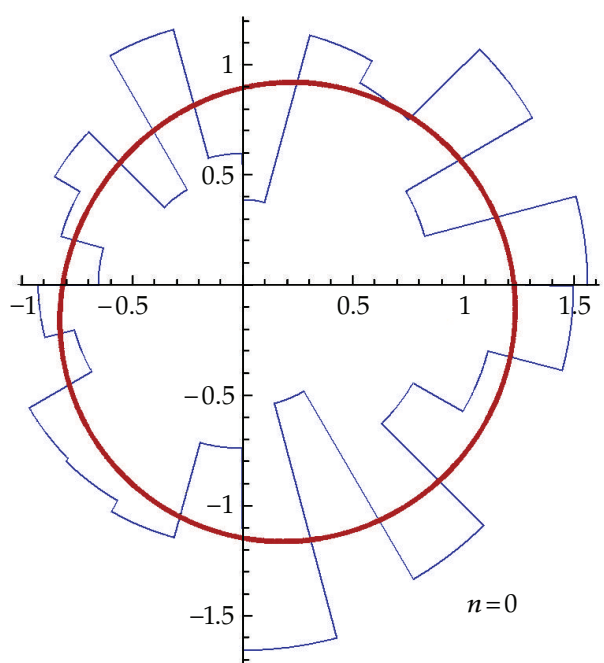

(a)

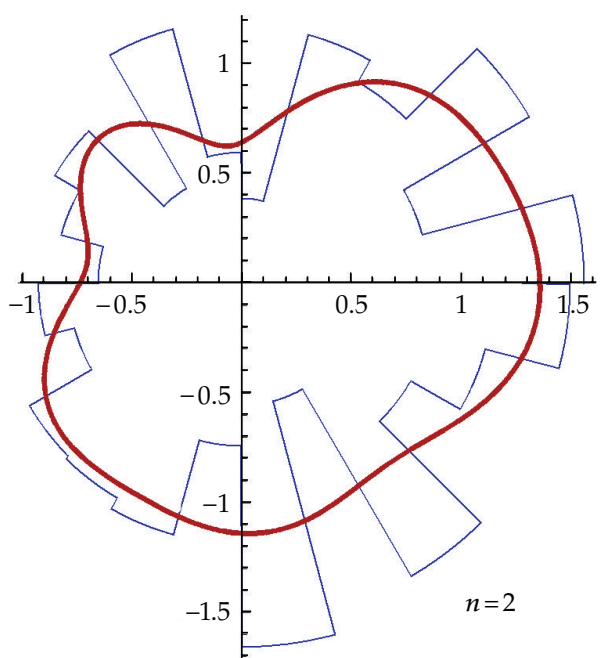

(c)

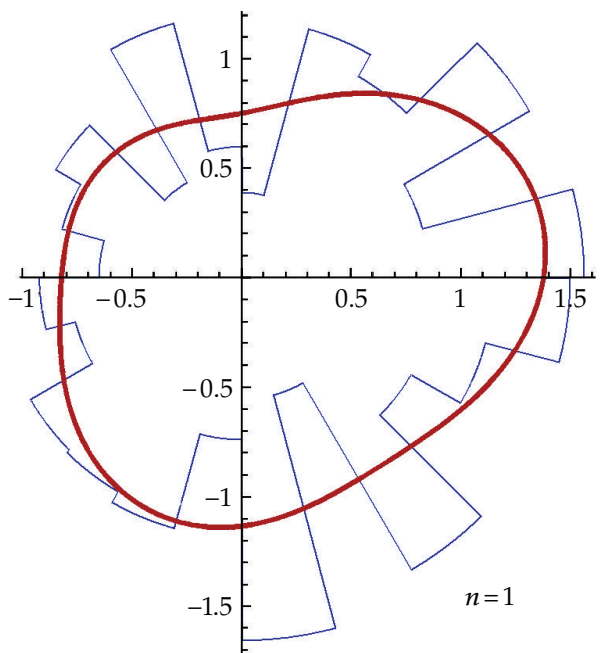

(b)

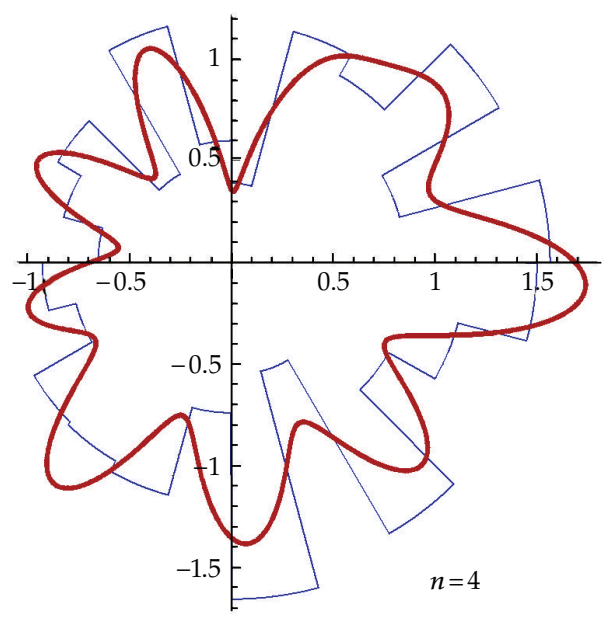

(d)

Figure 3: Original scalar-valued ODF sampling from $N\left(1.0,0.3^{2}\right)$ and its approximate ODFs using asymmetric characterization in 2D.

Note that $\delta_{\overline{m+2 n}}$ does not have the contracting property, that is, $\delta_{\overline{(m+2 n) \ominus 2}} \neq \delta_{\overline{m+2 n-2}}$, which means that the relationship between fabric tensors of different orders does hold for the symmetric characterization. Equation (4.4) looks somewhat confusing, so we just show its component form for $m=2$ and $n=1$,

$$
\delta_{\bar{i} \overline{\bar{p}}} \delta_{\bar{j} \overline{\bar{q}}} S_{i j k l} I_{\overline{k l} \overline{\overline{s t}}}=\alpha_{4} S_{\overline{p q s t}} .
$$

\subsection{Analytic Solution of $S_{m+2 n}$}

Likewise, our task is to find the analytic solution of $\mathbf{S}_{m+2 n}$ with respect to $\delta_{\overline{m+2 n}}$, or the inversed form of (4.4). 


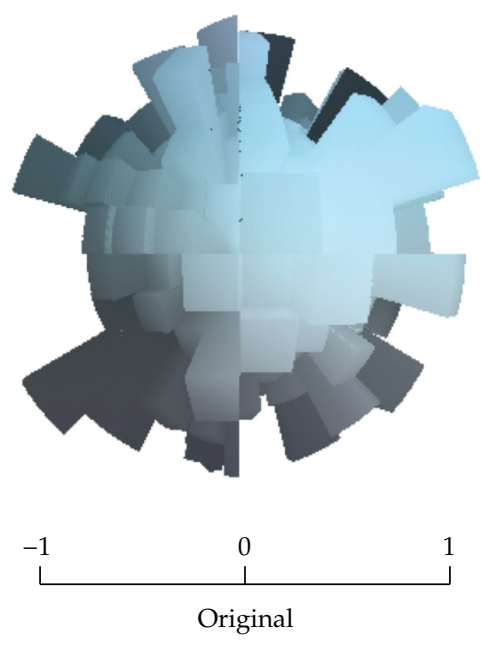

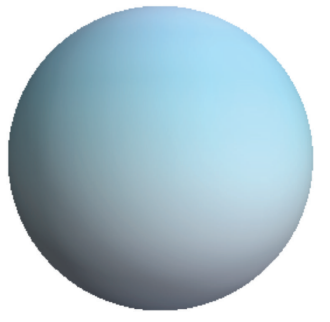

$n=0$

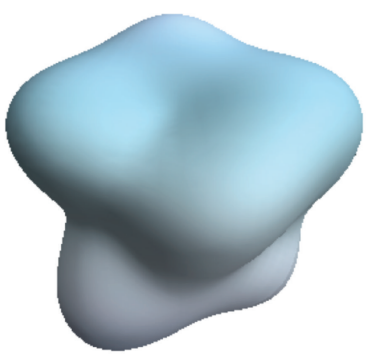

$n=2$

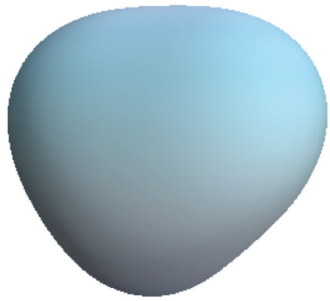

$n=1$

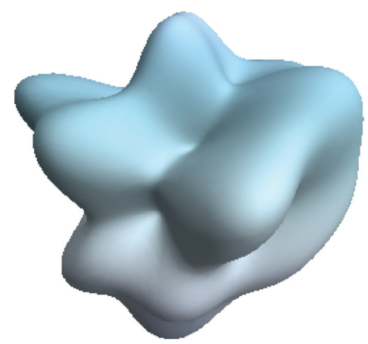

$n=3$

Figure 4: Original scalar-valued ODF sampling from $N\left(1.0,0.3^{2}\right)$ and its approximate ODFs using asymmetric characterization in 3D.

Known from (4.4), $\mathbf{S}_{m+2 n}$ is also a full symmetric tensor (just look at (4.6)). To avail of the orthogonal irreducible decomposition, the parity of $m$ must be discussed. We only consider the even case in the derivation. When $m$ is an even integer, let $m=2 p$, and the decomposition of $\mathbf{S}_{2 p+2 n}$ takes the following form:

$$
\mathbf{S}_{2 p+2 n}=\mathbf{S}_{\overline{2 p+2 n}} \stackrel{\text { def }}{=} \sum_{r=0}^{p+n} \boldsymbol{\Phi}_{\overline{2 r}} \bigotimes \mathbf{I}_{\overline{2 p+2 n-2 r}}
$$

where $\Phi_{\overline{2 r}}(r=0,1, \ldots, p+n)$ are accordingly termed the symmetrized fabric tensors of the third kind, of which any contractions vanish.

Applying (4.7) to (4.4), and after some manipulations, one obtains

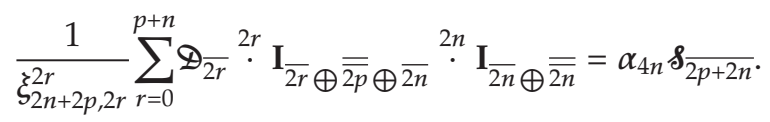

Note that each term in the summation takes the following recursive pattern (it is actually another more complicated ball and box puzzle),

$$
\boldsymbol{\Phi}_{\bar{a}}^{a} \cdot \mathbf{I}_{\bar{a} \oplus \overline{\bar{x}} \oplus \bar{b}} \cdot \mathbf{I}_{\bar{b} \oplus \overline{\bar{y}}}=\xi_{b+x, a}^{a} \sum_{l=0}^{x} \frac{\left(\begin{array}{c}
b \\
a-l
\end{array}\right)\left(\begin{array}{l}
x \\
l
\end{array}\right)}{\left(\begin{array}{c}
b+x \\
a
\end{array}\right)} \boldsymbol{\Phi}_{\overline{\bar{l}} \oplus \overline{a-l}} \stackrel{a-l}{\cdot} \mathbf{I}_{\overline{(a-l)} \oplus \overline{\overline{2 y}} \oplus \overline{\overline{b-(a-l)}}}{ }^{b-(a-l)} \mathbf{I}_{\overline{b-(a-l)}+\overline{\overline{x-l}}}
$$


Thus, (4.8) can be reduced to

$$
\sum_{r=0}^{p+n} \mu_{2 p, 2 n}^{2 r} \boldsymbol{\Phi}_{2 r} \bigotimes \mathbf{I}_{\overline{2 p+2 n-2 r}}=\varsigma_{\overline{2 p+2 n}}
$$

where the coefficient $\mu_{2 p, 2 n}^{2 r}$ is given by

$$
\mu_{2 p, 2 n}^{2 r}=\frac{F_{\text {recur }}(2 r, 2 n, 2 p, 2 n)}{\alpha_{4 n} \xi_{2 n+2 p, 2 r}^{2 r}}
$$

and $F_{\text {recur }}(a, b, x, y)$ is a recursive function drawn from (4.9), compatible for any parities of the parameters,

$$
F_{\text {recur }}(a, b, x, y)= \begin{cases}\alpha_{b+x} \sum_{l=0}^{b / 2} \frac{\xi_{y, b}^{b-2 l}}{\alpha_{b+x-2 l}}, & a=0, \\
\xi_{b+x, a}^{a} \sum_{l=0}^{x} \frac{\left(\begin{array}{c}
b \\
a-l
\end{array}\right)\left(\begin{array}{c}
x \\
l
\end{array}\right)}{\left(\begin{array}{c}
b+x \\
a
\end{array}\right)} F_{\text {recur }}(a-l, b-(a-l), y, x-l), & a>0 .\end{cases}
$$

The solving of (4.10) is through successive contraction and deviatorization, identical to that of (3.8), which yields the symmetrized fabric tensors of the third kind,

$$
\boldsymbol{\Phi}_{2 t}=\frac{1}{\mu_{2 p, 2 n}^{2 t} \zeta_{2 p+2 n, 2 t}^{2 t}} \delta_{\overline{\llbracket(2 p+2 n) \ominus(2 p+2 n-2 t) \rrbracket}}=\lambda_{2 p, 2 n}^{2 t} \boldsymbol{8}_{\llbracket(2 p+2 n) \ominus(2 p+2 n-2 t) \rrbracket} .
$$

For vector-valued ODFs $(m=1)$, the closed-form expression of the coefficient $\lambda_{1,2 n+1}^{2 t}$ can be shown as

$$
\lambda_{1,2 n+1}^{2 t}= \begin{cases}\frac{(2 n+2)^{2}}{(2 n+2)^{2}+2 t^{2}} \eta^{2 t}, & \text { in 2D; } \\ \frac{(2 n+2)^{2}}{(2 n+2)^{2}+2 t^{2}+2 t} \eta^{2 t}, & \text { in 3D. }\end{cases}
$$

However, for generic tensor-valued ODFs of higher orders, the closed-form expression of $\lambda$ is not found because of greater recursion depth.

The analytic solution of the symmetrized fabric tensors of the second kind is finally obtained, considering the parity of the approximation order,

$$
\begin{gathered}
\mathbf{S}_{2 p+2 n}=\sum_{t=0}^{p+n} \lambda_{2 p, 2 n}^{2 t} \sum_{r=0}^{t} \kappa_{2 t}^{r} \S_{\overline{(2 p+2 n) \ominus(2 p+2 n-2 t+2 r)}} \bigotimes \mathbf{I}_{2 p+2 n-2 t+2 r}, \\
\mathbf{S}_{2 p+1+2 n}=\sum_{t=0}^{p+n} \lambda_{2 p+1,2 n}^{2 t+1} \sum_{r=0}^{t} \kappa_{2 t+1}^{r} \S_{\overline{(2 p+1+2 n) \ominus(2 p+2 n-2 t+2 r)}} \bigotimes \mathbf{I}_{2 p+2 n-2 t+2 r} .
\end{gathered}
$$


The anticentrosymmetric tensor-valued $\operatorname{ODF} \mathbf{a} \bar{m}(\mathbf{n})$ is characterized by

$$
\mathbf{a}_{\bar{m}}(\mathbf{n}) \approx a_{\bar{m}}(\mathbf{n}) \stackrel{\text { def }}{=} \mathbf{A}_{\overline{m+2 n+1}} \stackrel{2 n+1}{\cdot} \mathbf{N}_{2 n+1}(\mathbf{n}),
$$

where the symmetrized fabric tensors of the second kind $\mathbf{A}_{\overline{m+2 n+1}}$ are solved as

$$
\begin{aligned}
& \mathbf{A}_{2 p+2 n+1}=\sum_{t=0}^{p+n} \lambda_{2 p, 2 n+1}^{2 t+1} \sum_{r=0}^{t} \kappa_{2 t+1}^{r} \mathcal{A} \frac{}{(2 p+2 n+1) \ominus(2 p+2 n-2 t+2 r)} \bigotimes \mathbf{I} \frac{}{2 p+2 n-2 t+2 r}, \\
& \mathbf{A}_{2 p+2 n+2}=\sum_{t=0}^{p+n+1} \lambda_{2 p+1,2 n+1}^{2 t} \sum_{r=0}^{t} \kappa_{2 t}^{r} \boldsymbol{\mathcal { A }} \frac{}{(2 p+2 n+2) \Theta(2 p+2 n+2-2 t+2 r)} \otimes \mathbf{I}_{2 p+2 n+2-2 t+2 r},
\end{aligned}
$$

with the symmetrized fabric tensors of the first kind defined as

$$
\mathcal{A} \frac{}{m+2 n+1} \stackrel{\text { def }}{=} \oint \mathbf{a}_{\bar{m}}(\mathbf{n}) \bigotimes \mathbf{N}_{\overline{2 n+1}}(\mathbf{n}) \mathrm{d} \mathbf{n} .
$$

The ultimately solved coefficients of the symmetrized fabric tensors of the second kind of lower orders for vector-valued ODFs and second-order tensor-valued ODFs are listed in Tables 2 and 3.

\subsection{Nonconvergence of the Symmetric Characterization}

The symmetric characterization is proved to be nonconvergent simply by a counter example. Assume an anticentrosymmetric vector-valued ODF $\mathbf{a}(\mathbf{n})=\left\{a_{i}(\mathbf{n})\right\}$ that is precisely determined by its second-order symmetrized fabric tensor,

$$
a_{i}(\mathbf{n})=a_{i}^{\mathrm{II}}(\mathbf{n})=A_{\overline{i r}} n_{r}
$$

As demanded by the convergence condition, $a_{i}(\mathbf{n})$ also must be precisely determined by its fourth-order symmetrized fabric tensor $A_{i j k l}$. Applying (4.19) to (4.18), one obtains the fourth-order fabric tensor of the first kind in $3 \mathrm{D}$,

$$
\mathcal{A}_{\overline{i j k l}}=\oint A_{\overline{i r}} n_{r} n_{j} n_{k} n_{l} \mathrm{~d} \mathbf{n}=\frac{1}{5} A_{\overline{i j}} \delta_{\overline{k l}},
$$

which is then substituted to $(4.17 \mathrm{~b})$ (or referring to the second row of the right half of Table 2) to determine the fourth-order fabric tensor of the second kind $A_{\overline{i j k l}}$,

$$
A_{\overline{i j k l}}=\frac{14}{11} A_{\overline{i j}} \delta_{\overline{k l}}-\frac{1}{11} A_{r r} \delta_{\overline{i j}} \delta_{\overline{k l}} .
$$

Finally, applying (4.21) to (4.16), one can find the convergence condition undermined because

$$
a_{i}^{\mathrm{IV}}(\mathbf{n})=A_{i j k l} n_{j} n_{k} n_{l}=\frac{7}{11} A_{\overline{i r}} n_{r}+\frac{7}{11} A_{\overline{r S}} n_{r} n_{s} n_{i}-\frac{1}{11} A_{\overline{r r}} n_{i} \neq a_{i}(\mathbf{n}) .
$$


Table 2: Coefficients of the lower order fabric tensors of the second kind using symmetric characterization for vector-valued ODFs (2D and 3D).

\begin{tabular}{lcccccccc}
\hline $\mathbf{S}_{\mathbf{1}}(\mathbf{n})$ & $\boldsymbol{S}_{1}$ & $\boldsymbol{s}_{\overline{1+2}}$ & $\boldsymbol{s}_{\overline{1+4}}$ & $\mathbf{a}_{\mathbf{1}}(\mathbf{n})$ & $\mathcal{A}_{0}$ & $\mathcal{A}_{\overline{1+1}}$ & $\mathcal{A}_{\overline{1+3}}$ & $\mathcal{A}_{\overline{1+5}}$ \\
\hline $\mathbf{S}_{1}$ & 1,1 & & & $\mathbf{A}_{\overline{1+1}}$ & 0,0 & 2,3 & & \\
$\mathbf{S}_{\overline{1+2}}$ & $-\frac{6}{5},-\frac{45}{22}$ & $4, \frac{15}{2}$ & & $\mathbf{A}_{\overline{1+3}}$ & $\frac{2}{5}, \frac{15}{22}$ & $-\frac{24}{5},-\frac{105}{11}$ & $8, \frac{35}{2}$ & \\
$\mathbf{S}_{\overline{1+4}}$ & $\frac{555}{221}, \frac{13475}{2664}$ & $-\frac{240}{17},-\frac{4725}{148}$ & $16, \frac{315}{8}$ & $\mathbf{A}_{\overline{1+5}}$ & $-\frac{27}{65},-\frac{95}{112}$ & $\frac{684}{65}, \frac{675}{28}$ & $-\frac{480}{13},-\frac{1485}{16}$ & $32, \frac{693}{8}$ \\
\hline
\end{tabular}

Table 3: Coefficients of the lower order fabric tensors of the second kind using symmetric characterization for second-order tensor-valued ODFs (2D and 3D).

\begin{tabular}{lcccc}
\hline $\mathbf{S}_{\overline{2}}(\mathbf{n})$ & $\boldsymbol{s}_{0}$ & $\boldsymbol{s}_{\overline{2}}$ & $\boldsymbol{s}_{\overline{2+2}}$ & $\boldsymbol{s}_{\overline{2+4}}$ \\
\hline $\mathbf{S}_{\overline{2}}$ & 0,0 & 1,1 & \\
$\mathbf{S}_{\overline{2+2}}$ & $\frac{1}{15}, \frac{45}{418}$ & $-\frac{4}{3}, \frac{45}{19}$ & $4, \frac{15}{2}$ & \\
$\mathbf{S}_{\overline{2+4}}$ & $-\frac{101}{598},-\frac{44975}{141696}$ & $\frac{98}{23}, \frac{23625}{2624}$ & $-16,-\frac{4725}{128}$ & $16, \frac{315}{8}$ \\
\hline$a_{2}(\mathbf{n})$ & $\mathcal{A}_{1}$ & $\mathcal{A}_{\overline{2+1}}$ & $\mathcal{A}_{\overline{2+3}}$ & $\boldsymbol{A}_{\overline{2+5}}$ \\
\hline $\mathbf{A}_{\overline{2+1}}$ & 0,0 & 2,3 & & \\
$\mathbf{A}_{\overline{2+3}}$ & $\frac{135}{154}, \frac{4725}{2914}$ & $-\frac{60}{11},-\frac{525}{47}$ & $8, \frac{35}{2}$ & \\
$\mathbf{A}_{\overline{2+5}}$ & $-\frac{77336}{53001},-\frac{32007339}{10178960}$ & $\frac{63980}{4077}, \frac{858627}{23134}$ & $-\frac{1120}{27},-\frac{72765}{688}$ & $32, \frac{693}{8}$ \\
\hline
\end{tabular}

Despite of such non-convergence, the symmetric characterization is still available for most irregular tensor-valued ODFs. On one hand, the approximation condition and accuracy are definitely determined for a given order, while the accuracy may well rise with the used order owing to more independent components in the fabric tensors, as illustrated in the next subsection. On the other hand, if full symmetry is prescribed on the fabric tensors, the symmetric characterization is of the highest accuracy, particularly much more accurate than the ungrounded direct symmetrization of the asymmetric fabric tensors.

\subsection{Accuracy Analysis}

Accuracy analyses are performed on vector-valued and second-order tensor-valued ODFs, because the symmetric characterization is identical to the asymmetric one for scalar-valued ODFs. The original vector-valued ODF $\mathbf{v}(\mathbf{n})$ is generated in the following way: the magnitude $|\mathbf{v}|(\mathbf{n})$ samples from $N\left(1.0, \sigma^{2}\right)$, with $\sigma$ ranging from 0.1 to 0.5 , and the stereo angles $\theta_{\mathbf{v}}(\mathbf{n})$ and $\phi_{\mathbf{v}}(\mathbf{n})$ from $N\left(\theta_{\mathbf{n}},(\sigma \pi / 2)^{2}\right)$ and $N\left(\phi_{\mathbf{n}},(\sigma \pi / 2)^{2}\right)$, respectively. The second-order tensor-valued ODF $\mathbf{m}_{\overline{2}}(\mathbf{n})$ is formed by $\mathbf{v} \otimes \mathbf{v} /|\mathbf{v}|$. Both the asymmetric and symmetric characterizations are investigated and compared.

The results indicate that the symmetric characterization still follows the general rule of the asymmetric one; that is to say, the fitness majorly decreases with the dispersion degree of the original data $(\sigma)$ and improves only when the approximation order reaches very high. So we only display and discuss the results for $\sigma=0.3$. The squared error ratios of the two characterizations in 2D and 3D are shown in Figures 5 and 6, respectively. 


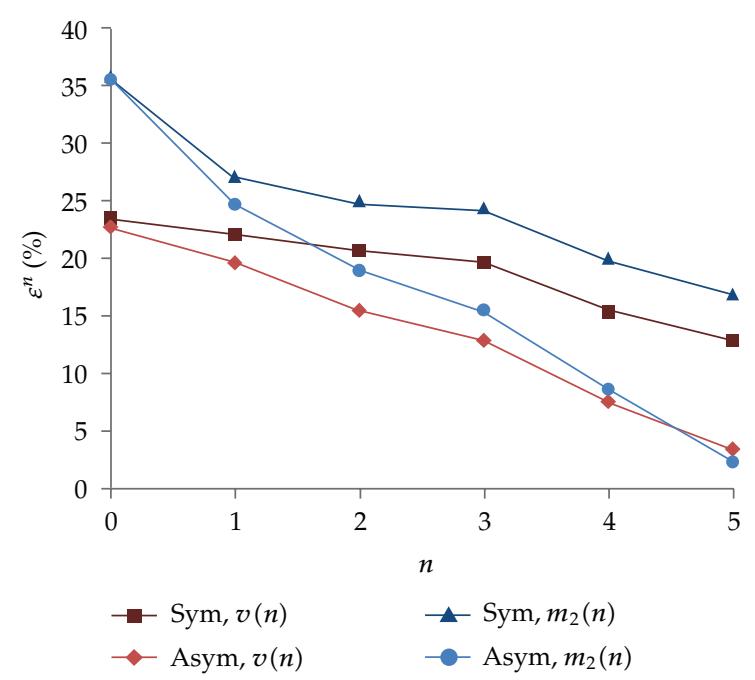

Figure 5: Squared error ratios of symmetric and asymmetric characterizations of vector-valued and secondorder tensor-valued ODFs sampling from $N\left(1.0,0.3^{2}\right)$ in 2D.

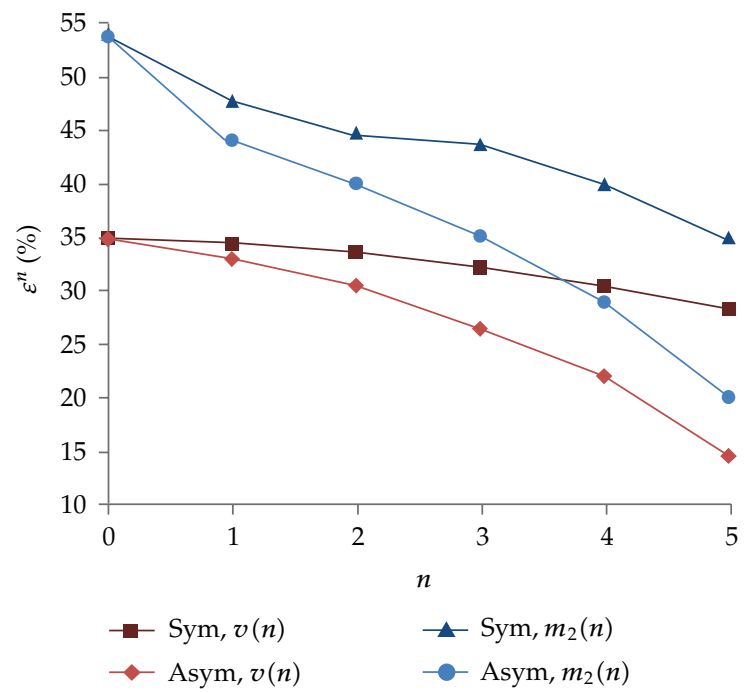

Figure 6: Squared error ratios of symmetric and asymmetric characterizations of vector-valued and secondorder tensor-valued ODFs sampling from $N\left(1.0,0.3^{2}\right)$ in $3 \mathrm{D}$.

Known from Figures 5 and 6, the accuracy and convergence rate of the symmetric characterization are lower than those of the asymmetric one, because of fewer independent components of cause. But the difference does not reach an unacceptable degree, especially for lower orders. Therefore, the symmetric characterization is by all means an available solution for where fabric tensors are required in full symmetry; after all, it is the most accurate way to obtain full symmetric fabric tensors. The original and approximate vector-valued ODFs related to $N\left(1.0,0.3^{2}\right)$ in $2 \mathrm{D}$ and $3 \mathrm{D}$ are plotted in Figures 7 and 8 , respectively, where the 


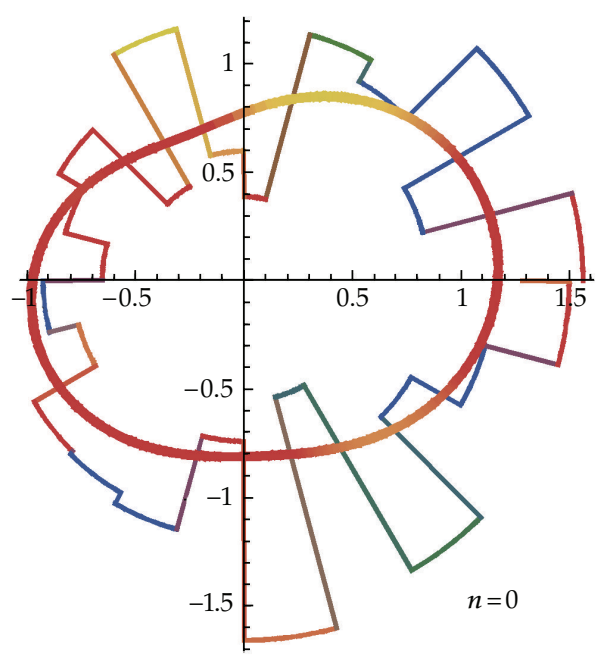

(a)

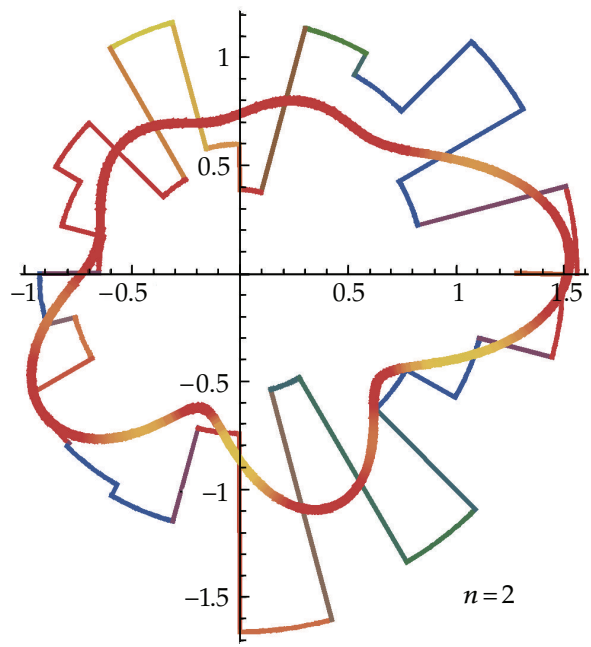

(c)

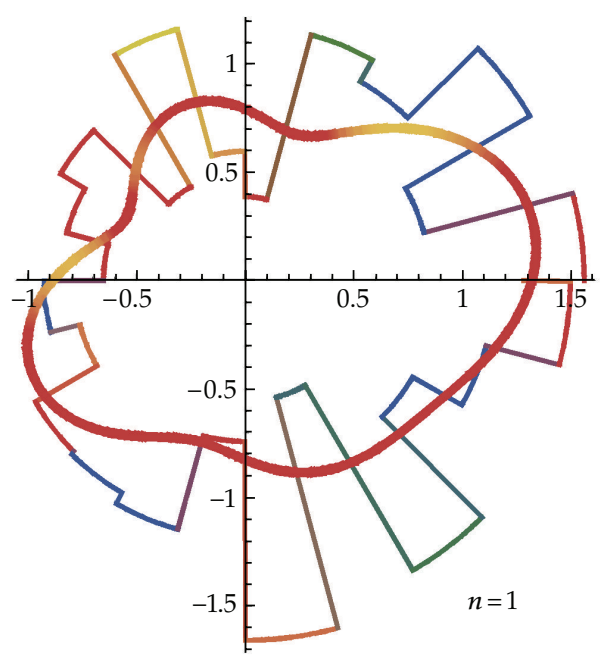

(b)

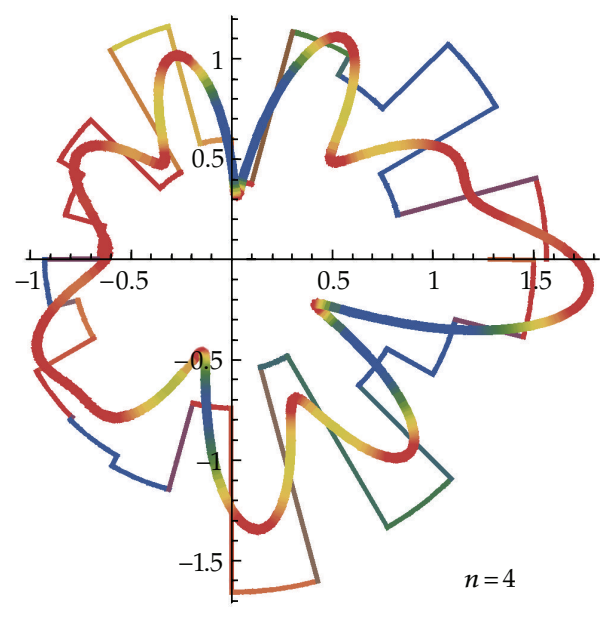

(d)

Figure 7: Original vector-valued ODF sampling from $N\left(1.0,0.3^{2}\right)$ and its approximate ODFs using symmetric characterization in 2D.

magnitude $|\mathbf{v}|$ and the cosine of the deviation angle $\cos \langle\mathbf{v}, \mathbf{n}\rangle$ serve as the radius and color functions, respectively.

\section{Application: Microplane-Based Damage Effective Stress}

In this section, we show an application of tensor-valued ODF and fabric tensor in continuum damage mechanics, which helps to clarify the multiaxial generalization of the damage effective stress in the classical models. Continuum damage mechanics deals with the degradation of solid materials due to small defects (voids, microcracks, and dislocations) using continuity modeling. Kachanov [27] and Rabotnov [28] raised the concept of damage effective stress, which constitutes the core of continuum damage mechanics. 


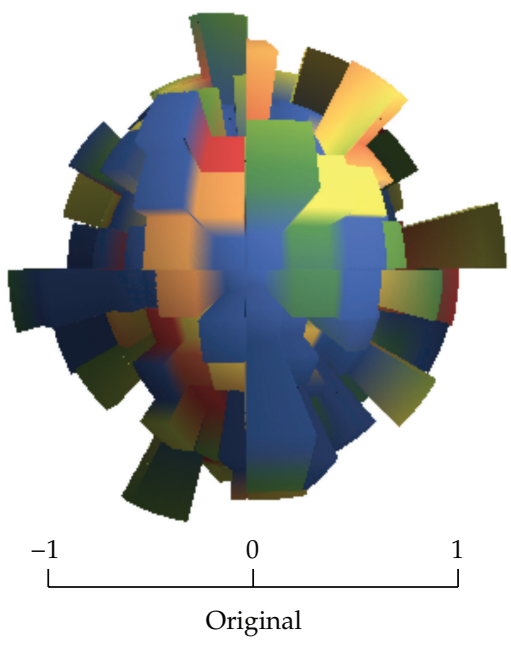

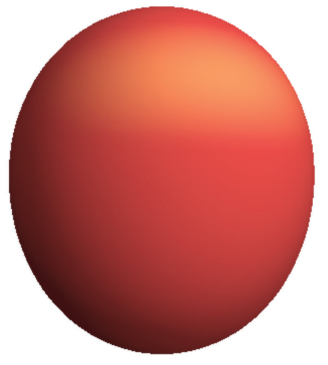

$n=0$

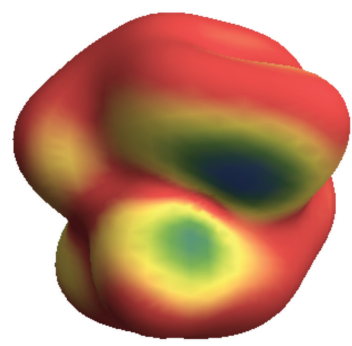

$n=2$

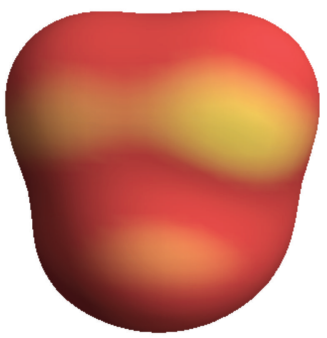

$n=1$

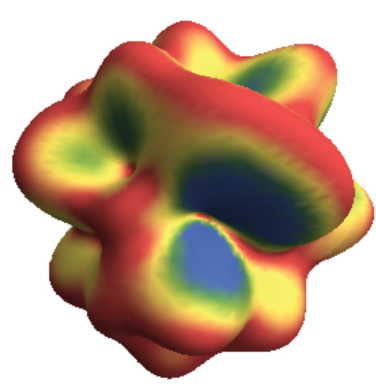

$n=3$

Figure 8: Original vector-valued ODF sampling from $N\left(1.0,0.3^{2}\right)$ and its approximate ODFs using symmetric characterization in 3D.

We start from the original concept of uniaxial damage. Consider a smooth tensile bar of nominal cross-sectional area $S$ subjected to a uniaxial stress $\sigma$. The uniaxial damage effective stress $\bar{\sigma}$ is defined by Kachanov [27] and Rabotnov [28]:

$$
\bar{\sigma}=\frac{\sigma}{(1-D)}, \quad D=\frac{S_{D}}{S}
$$

where $D$ is the scalar damage variable and $S_{D}$ the damaged cross-sectional area.

The multiaxial generalization of the Kachanov-Rabotnov damage effective stress is not a straightforward task, especially for anisotropic damage effects. Quite a few models have been developed, as summarized by Zheng and Betten [29] into five types. All these models start from a subjective premise that the damage effective stress is a Cauchy-like stress tensor of order two, which is critically reviewed by Yang et al. [7, 23], who proposed that damage effective stress should be generalized as a vector-valued ODF owing to its microplane-based nature.

A microplane is a plane of any orientation cutting the material at a given point, which is represented by the unit normal of that orientation, $\mathbf{n}=\left\{n_{i}\right\}$. The stress state on a microplane $\mathbf{n}$ is described by the Cauchy stress ODF $\sigma_{i}=\sigma_{i}(\mathbf{n})$, which is precisely determined by the second-order Cauchy stress tensor $\sigma_{i j}$,

$$
\sigma_{i}=\sigma_{i}(\mathbf{n}) \stackrel{\text { def }}{=} \sigma_{i j} n_{j} .
$$


Equation (5.1) can be naturally extended onto each microplane to define the damage effective stress ODF $\bar{\sigma}_{i}(\mathbf{n})$, with anisotropic damage effects measured by a scalar-valued ODF $D(\mathbf{n})$,

$$
\bar{\sigma}_{i}(\mathbf{n}) \stackrel{\text { def }}{=} \frac{\sigma_{i}(\mathbf{n})}{1-D(\mathbf{n})}=\frac{\sigma_{i j}}{1-D(\mathbf{n})} n_{j} \approx \bar{\sigma}_{i j} n_{j}
$$

Obviously, because the term $\sigma_{i j} /[1-D(\mathbf{n})]$ is nontensorial, the second-order tensor $\bar{\sigma}_{i j}$ cannot fully describe the damage effective stress $\operatorname{ODF} \bar{\sigma}_{i}(\mathbf{n})$ but is only an approximate estimation. This is the essential reason why the definition of a second-order damage effective stress tensor is so controversial; see, for example, the discussion by Zheng and Betten [29], Kachanov [30], and Lemaitre et al. [31].

In fact, the second-order tensor $\bar{\sigma}_{i j}$ can be identified with the second-order fabric tensor of the vector-valued ODF $\bar{\sigma}_{i}(\mathbf{n})$, and of course, the order can reach higher than two. Because only the symmetric part of $\bar{\sigma}_{i j}$ accounts for constitutive equations [32-34], the symmetric characterization should be applied. For example, the second- and fourth-order symmetrized fabric tensors of $\bar{\sigma}_{i}(\mathbf{n})$ in $3 \mathrm{D}$ are given by

$$
\begin{gathered}
\bar{\sigma}_{i j}=3 \widetilde{\sigma}_{i j}, \\
\bar{\sigma}_{i j k l}=\frac{35}{2} \widetilde{\sigma}_{i j k l}-\frac{105}{11} \widetilde{\sigma}_{r r i \bar{j}} \delta_{\overline{k l}}+\frac{15}{22} \widetilde{\sigma}_{r r s s} \delta_{i j} \delta_{\overline{k l}},
\end{gathered}
$$

where $\tilde{\sigma}_{i j}$ and $\tilde{\sigma}_{i j k l}$ are the symmetrized fabric tensors of the first kind,

$$
\begin{gathered}
\tilde{\sigma}_{i j} \stackrel{\text { def }}{=} \oint \bar{\sigma}_{\bar{i}} n_{\bar{j}} \mathrm{~d} \mathbf{n}, \\
\widetilde{\sigma}_{i j k l} \stackrel{\text { def }}{=} \oint \bar{\sigma}_{\bar{i}} n_{\bar{j}} n_{\bar{k}} n_{\bar{l}} \mathrm{~d} \mathbf{n} .
\end{gathered}
$$

Substituting (5.3) to (5.5a) and then to (5.4a), the second-order fabric tensor $\bar{\sigma}_{i j}$ is finally obtained,

$$
\bar{\sigma}_{i j}=\frac{1}{2}\left(\Psi_{i k} \sigma_{k j}+\sigma_{i k} \Psi_{k j}\right), \quad \text { where } \Psi_{i j}=3 \oint \frac{1}{1-D(\mathbf{n})} n_{i} n_{j} \mathrm{~d} \mathbf{n} .
$$

This is just the anisotropic damage model proposed by Murakami [34].

However, the definition of the damage effective stress ODF $\bar{\sigma}_{i}(\mathbf{n})$ in (5.3) is quite narrow, which only deals with coaxial damage effects; that is, damage only affects the net area of each microplane without changing its orientation. In general, noncoaxial damage effects should be described by a second-order tensor-valued ODF $M_{i j}(\mathbf{n})$, which connects the damage effective stress ODF $\bar{\sigma}_{i}(\mathbf{n})$ to the Cauchy stress ODF $\sigma_{i}(\mathbf{n})$ on each microplane with full geometry equivalence. In noncoaxial cases, it can be shown that if the second-order fabric tensor $\bar{\sigma}_{i j}$ is applied to characterize $\bar{\sigma}_{i}(\mathbf{n})$, the widely applied anisotropic model based on the fourth-order damage effect tensor $[16,29,32,33,35,36]$ in Voigt symmetry can be realized. The noncoaxial cases as well as microplane-based physics equivalence hypotheses and damage evolution laws will be elaborated in another paper, which may help to clarify some uncertainties over the basic concepts of continuum damage mechanics. 


\section{Conclusions}

The fabric tensor approach for the largest generality of tensor-valued orientation distribution functions (ODFs) is presented, including the analytical solution and accuracy analysis of the asymmetric and symmetric characterizations. The asymmetric characterization of tensorvalued ODFs is in complete parallel to that of scalar-valued ODFs by Kanatani [5], where the components of tensor-valued ODFs are handled as independent scalar-valued ODFs. The asymmetric characterization proves to be convergent [5] and featured by the relationship of fabric tensors of different orders [7]. The symmetric characterization does not possess such convergence property and relationship, but is still an available solution for most irregular tensor-valued ODFs whose fabric tensors are prescribed in full symmetry. Accuracy analyses are performed on normally distributed random ODFs to evaluate the approximation quality of the two characterizations. The results indicate that the fitness is dominated by the dispersion degree of the original ODFs rather than the orders of the fabric tensors. The accuracy and convergence rate of the symmetric characterization are lower than those of the asymmetric one, but the differences keep in an acceptable range for lower approximation orders.

\section{Acknowledgments}

This work is supported by the National Natural Science Foundation of China under Projects 50925931 and 11172150 and subsidized by the special funds for major state basic research projects with Grant no. 2009CB724604.

\section{References}

[1] K. V. Mardia, "Statistics of directional data," Journal of the Royal Statistical Society Series B, vol. 37, no. 3, pp. 349-393, 1975.

[2] P. E. Jupp and K. V. Mardia, "A unified view of the theory of directional statistics," International Statistical Review, vol. 57, pp. 261-294, 1989.

[3] H. J. Bunge, "Fabric analysis by orientation distribution functions," Tectonophysics, vol. 78, no. 1-4, pp. 1-21, 1981.

[4] J. H. Bunge, Texture Analysis in Materials Science: Mathematical Methods, Butterworths, London, UK, 1982.

[5] K. Kanatani, "Distribution of directional data and fabric tensors," International Journal of Engineering Science, vol. 22, no. 2, pp. 149-164, 1984.

[6] S. G. Advani and C. L. Tucker III, "The use of tensors to describe and predict fiber orientation in short fiber composites," Journal of Rheology, vol. 31, pp. 751-784, 1987.

[7] Q. Yang, X. Chen, and W. Y. Zhou, "Effective stress and vector-valued orientational distribution functions," International Journal of Damage Mechanics, vol. 17, no. 2, pp. 101-121, 2008.

[8] Y. F. Dafalias, "Orientation distribution function in non-affine rotations," Journal of the Mechanics and Physics of Solids, vol. 49, no. 11, pp. 2493-2516, 2001.

[9] Y. Rathi, O. Michailovich, M. E. Shenton, and S. Bouix, "Directional functions for orientation distribution estimation," Medical Image Analysis, vol. 13, no. 3, pp. 432-444, 2009.

[10] S. C. Cowin, "Anisotropic poroelasticity: fabric tensor formulation," Mechanics of Materials, vol. 36, no. 8, pp. 665-677, 2004.

[11] J. Rahmoun, D. Kondo, and O. Millet, "A 3D fourth order fabric tensor approach of anisotropy in granular media," Computational Materials Science, vol. 46, no. 4, pp. 869-880, 2009.

[12] X. Li and H. S. Yu, "Tensorial characterisation of directional data in micromechanics," International Journal of Solids and Structures, vol. 48, no. 14-15, pp. 2167-2176, 2011.

[13] M. Oda, "Fabric tensors for discontinuous geological materials," Soils and Foundations, vol. 22, no. 4, pp. 96-108, 1982. 
[14] V. A. Lubarda and D. Krajcinovic, "Damage tensors and the crack density distribution," International Journal of Solids and Structures, vol. 30, no. 20, pp. 2859-2877, 1993.

[15] Q. Yang, Z. Li, and L. G. Tham, "An explicit expression of second-order fabric-tensor dependent elastic compliance tensor," Mechanics Research Communications, vol. 28, no. 3, pp. 255-260, 2001.

[16] G. Z. Voyiadjis and P. I. Kattan, "Damage mechanics with fabric tensors," Mechanics of Advanced Materials and Structures, vol. 13, no. 4, pp. 285-301, 2006.

[17] J. Ning and E. C. Aifantis, "Anisotropic yield and plastic flow of polycrystalline solids," International Journal of Plasticity, vol. 12, no. 10, pp. 1221-1240, 1996.

[18] P. D. Wu and E. Van Der Giessen, "On improved network models for rubber elasticity and their applications to orientation hardening in glassy polymers," Journal of the Mechanics and Physics of Solids, vol. 41, no. 3, pp. 427-456, 1993.

[19] J. H. Kinney, J. S. Stölken, T. S. Smith, J. T. Ryaby, and N. E. Lane, “An orientation distribution function for trabecular bone," Bone, vol. 36, no. 2, pp. 193-201, 2005.

[20] M. Parsheh, M. L. Brown, and C. K. Aidun, "Investigation of closure approximations for fiber orientation distribution in contracting turbulent flow," Journal of Non-Newtonian Fluid Mechanics, vol. 136 , no. 1, pp. 38-49, 2006.

[21] K. Tabelow, H. U. Voss, and J. Polzehl, “Modeling the orientation distribution function by mixtures of angular central Gaussian distributions," Journal of Neuroscience Methods, vol. 203, no. 1, pp. 200-211, 2012.

[22] Q. Yang, X. Chen, and L. G. Tham, "Relationship of crack fabric tensors of different orders," Mechanics Research Communications, vol. 31, no. 6, pp. 661-666, 2004.

[23] Q. Yang, X. Chen, and W. Y. Zhou, "Microplane-damage-based effective stress and invariants," International Journal of Damage Mechanics, vol. 14, no. 2, pp. 179-191, 2005.

[24] Z. P. Bažant and P. G. Gambarova, "Crack shear in concrete: crack band microplane model," Journal of Structural Engineering, vol. 110, no. 9, pp. 2015-2035, 1984.

[25] I. Carol and Z. P. Bažant, "Damage and plasticity in microplane theory," International Journal of Solids and Structures, vol. 34, no. 29, pp. 3807-3835, 1997.

[26] Q.-S. Zheng and W.-N. Zou, "Irreducible decompositions of physical tensors of high orders," Journal of Engineering Mathematics, vol. 37, no. 1-3, pp. 273-288, 2000.

[27] L. M. Kachanov, "Time of the Rupture Process under Creep Conditions," Izvestiya Sibirskogo Otdeleniya,. Akademii Nauk SSSR., Seriya Tekhnicreskikh Nauk, vol. 8, pp. 26-31, 1958.

[28] I. N. Rabotnov, "On the equations of state for creep," Progress in Applied Mechanics, the Prager Anniversary Volume, vol. 8, pp. 307-315, 1963.

[29] Q. S. Zheng and J. Betten, "On damage effective stress and equivalence hypothesis," International Journal of Damage Mechanics, vol. 5, no. 3, pp. 219-240, 1996.

[30] L. M. Kachanov, "Effective elastic properties of cracker solids: critical review of some basic concepts," Applied Mechanics Review, vol. 45, no. 8, pp. 304-335, 1992.

[31] J. Lemaitre, R. Desmorat, and M. Sauzay, "Anisotropic damage law of evolution," European Journal of Mechanics, A/Solids, vol. 19, no. 2, pp. 187-208, 2000.

[32] G. Z. Voyiadjis and P. I. Kattan, "On the symmetrization of the effective stress tensor in continuum damage mechanics," Journal of the Mechanical Behavior of Materials, vol. 7, pp. 139-165, 1996.

[33] G. Z. Voyiadjis and T. Park, "Anisotropic damage effect tensors for the symmetrization of the effective stress tensor," Journal of Applied Mechanics, Transactions ASME, vol. 64, no. 1, pp. 106-110, 1997.

[34] S. Murakami, "Mechanical modeling of material damage," Journal of Applied Mechanics, Transactions ASME, vol. 55, no. 2, pp. 280-286, 1988.

[35] J. L. Chaboche, "Continuous damage mechanics-a tool to describe phenomena before crack initiation," Nuclear Engineering and Design, vol. 64, no. 2, pp. 233-247, 1981.

[36] D. Krajcinovic, “Continuous damage mechanics," Applied Mechanics Review, vol. 37, pp. 1-6, 1984. 


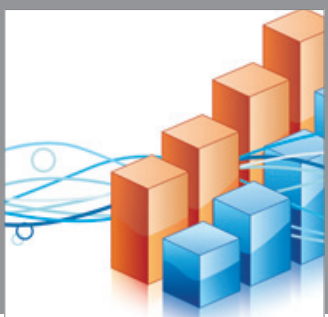

Advances in

Operations Research

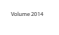

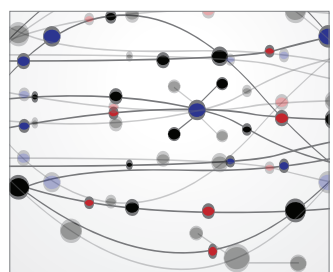

\section{The Scientific} World Journal
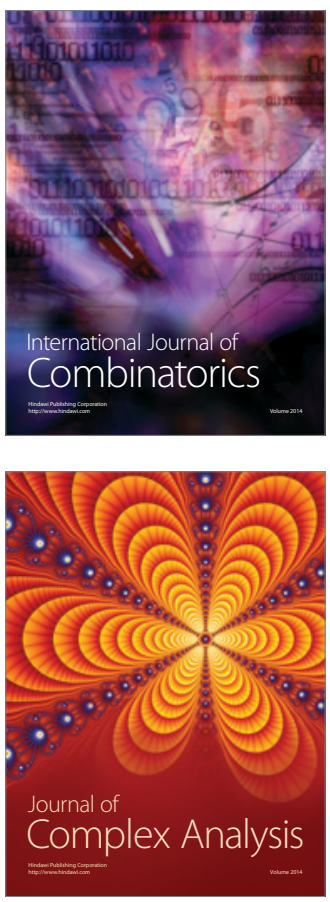

International Journal of

Mathematics and

Mathematical

Sciences
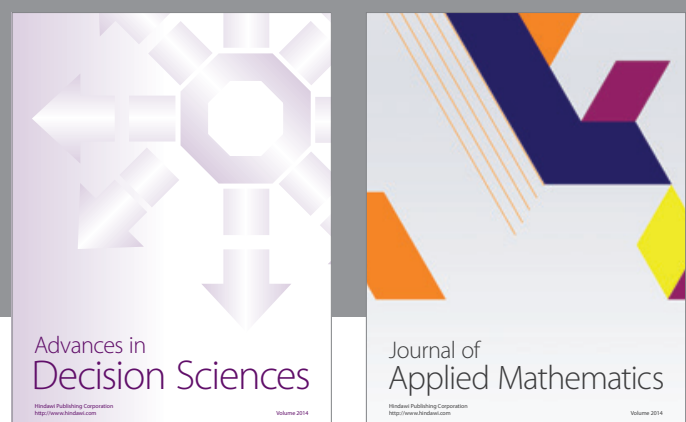

Journal of

Applied Mathematics
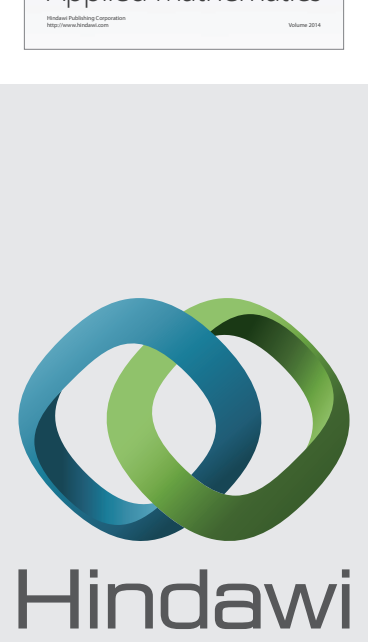

Submit your manuscripts at http://www.hindawi.com
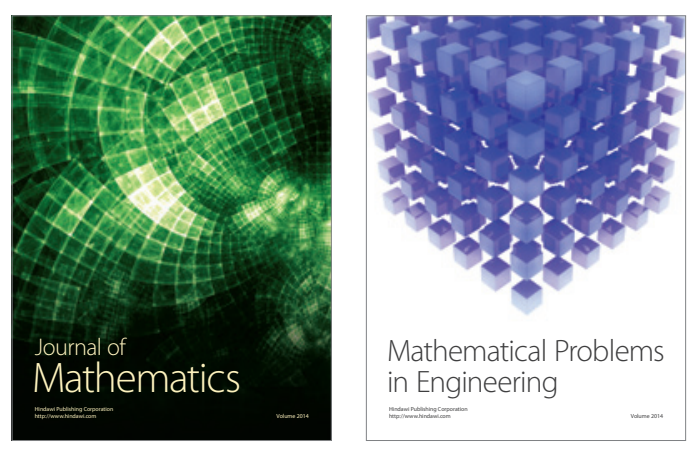

Mathematical Problems in Engineering
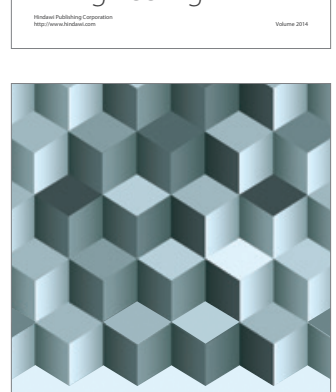

Journal of

Function Spaces
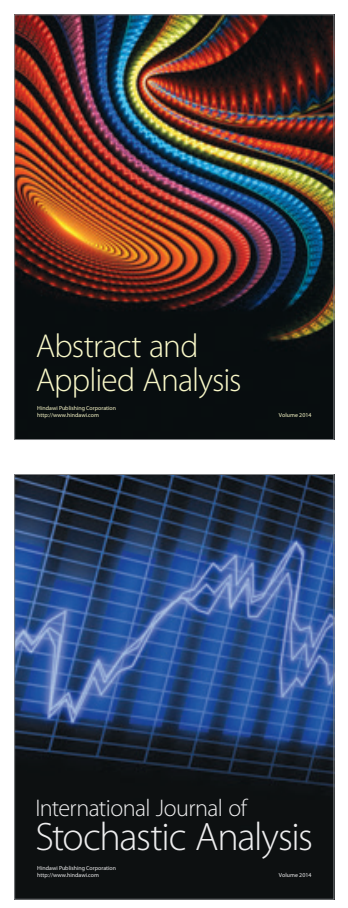

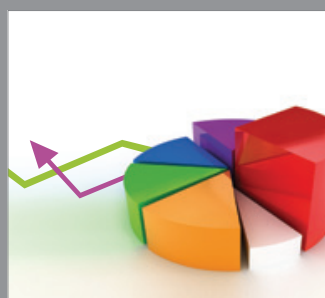

ournal of

Probability and Statistics

Promensencen
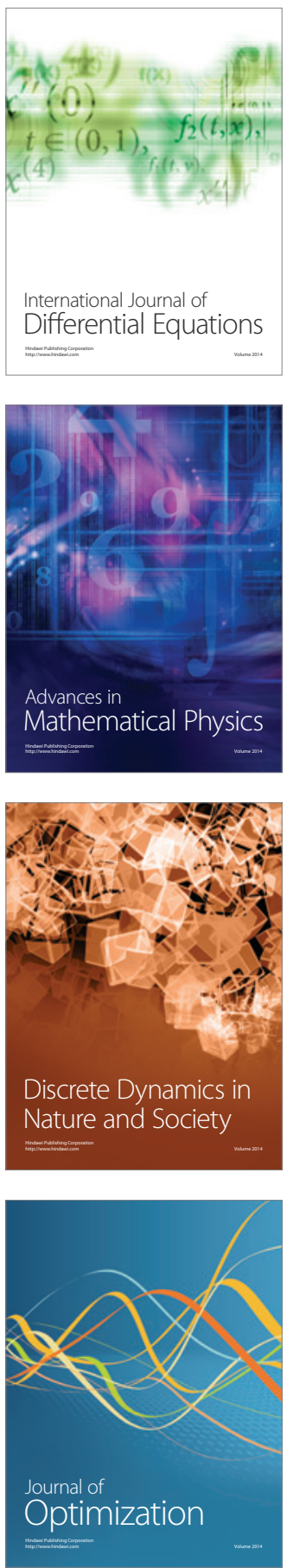